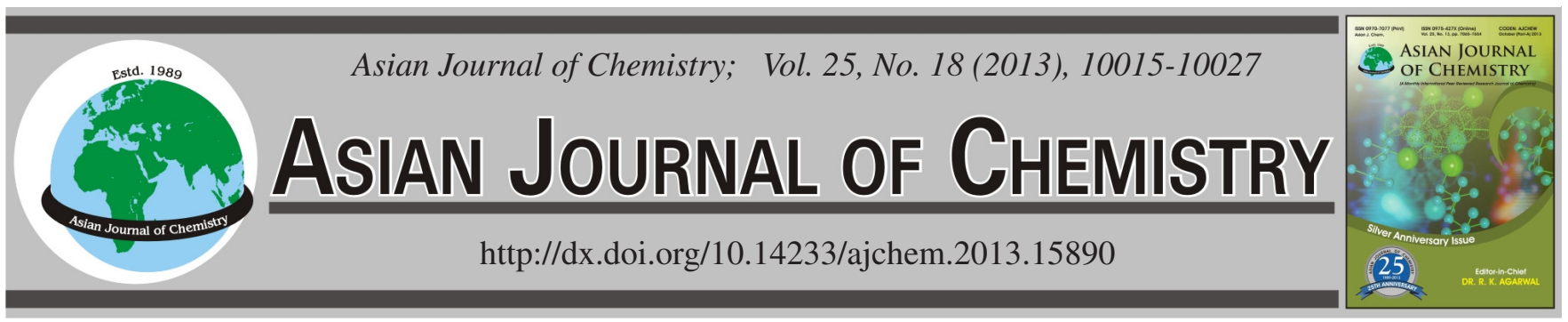

\title{
REVIEW
}

\section{Aporphine Alkaloids: A Kind of Alkaloids' Extract Source, Chemical Constitution and Pharmacological Actions in Different Botany}

Jianxin $\mathrm{Chen}^{\dagger}$, Kuo GaO ${ }^{\dagger}$, Tiegang Liu ${ }^{\dagger}$, Huinui Zhao, Juan Wang, Hao Wu, Bing Liu and Wei Wang*

Beijing University of Chinese Medicine, 11 Bei San Huan Dong Lu, ChaoYang District, Beijing 100029, P.R. China

*Corresponding author: Fax: +86 10 64286283; Tel: +86 10 64286508; E-mail: wangwei@ bucm.edu.cn

$\dagger$ These authors contributed equally to this work.

\begin{abstract}
As a kind of common compositions in plants, there are many researches about alkaloids, which was firstly found from Nelumbo nucifera. Now more and more aporphine alkaloids appear during the experiments, which belong to isoquinoline type. There are number of pesticide effects about them, which have been revealed in recent years. So comprehensive information about the locality, formation and pesticide effects have been studied and presented in this research in different botany about aporphine alkaloids.

Key Words: Alkaloids' extract, Chemical constitution, Pharmacological actions, Aporphine alkaloids, Nelumbo nucifera.
\end{abstract}

\section{INTRODUCTION}

Studies of the alkaloids of Nelumbo nucifera began in the 19th century. Since Nelumbo nucifera alkali (nuciferine), Ogo the methyl nuciferine (O-nornuciferine) and Lin alkali (roemerine) had been found from Nelumbo nucifera, so far a number of alkaloid compounds have been isolated from Nelumbo nucifera. The Nelumbo nucifera alkaloids, based on the nucleus structure, are divided into three categories i.e., single benzyl isoquinoline, aporphine and dehydrogenase aporphine.

Single benzyl isoquinoline e.g., armepavine, N-methylisococlaurine, N-methylcoclaurine. Structures of compounds are shown in Fig. 1 and Table-1.<smiles>[R6]Oc1cc2c(cc1O[R6])[C@@H](Cc1ccc(O)cc1)N(C)CC2</smiles>

Fig. 1. Nucleus structures of single benzyl isoquinoline

\begin{tabular}{cccc}
\multicolumn{5}{c}{ TABLE-1 } \\
STRUCTURES OF SUBSTITUENT GROUPS IN \\
\multicolumn{4}{c}{ SINGLE BENZYL ISOQUINOLINE } \\
\hline Compound & $\mathrm{R}_{1}$ & $\mathrm{R}_{2}$ & Name \\
\hline Armepavine & $\mathrm{CH}_{3}$ & $\mathrm{CH}_{3}$ & Armepavine \\
N-Methylisococlaurine & $\mathrm{H}$ & $\mathrm{CH}_{3}$ & N-Methylisococlaurine \\
N-Methylcoclaurine & $\mathrm{CH}_{3}$ & $\mathrm{H}$ & N-Methylcoclaurine \\
\hline
\end{tabular}

Aporphine: Nuciferine, N-nornuciferine, O-nornuciferine, anonaine, roemerine, liriodenine, $\mathrm{N}$-norarmepavine, 2-hydroxy1-methoxyaporphine. Structures of compounds are shown in Figs. 2 and 3; Table-2.<smiles>[R]c1cc2c3c(c1[R2])-c1ccccc1CC3N([R])CC2</smiles>

Fig. 2. Nucleus structures of aporphine<smiles>O=C1c2ccccc2-c2c3c(cc4ccnc1c24)OCO3</smiles>

Fig. 3. Structures of liriodenine

Dehydrogenase aporphine: Dehydronuciferinene, dehydroroemerine. Structures of compounds are shown in Fig. 4 and Table-3. 
TABLE-2

STRUCTURES OF SUBSTITUENT GROUPS IN APORPHINE

\begin{tabular}{|c|c|c|c|c|}
\hline Compound & $\mathrm{R}_{1}$ & $\mathrm{R}_{2}$ & $\mathrm{R}_{3}$ & Name \\
\hline Nuciferine & $\mathrm{OCH}_{3}$ & $\mathrm{OCH}_{3}$ & $\mathrm{CH}_{3}$ & Nuciferine \\
\hline $\mathrm{N}$-Nornuciferine & $\mathrm{OCH}_{3}$ & $\mathrm{OCH}_{3}$ & $\mathrm{H}$ & N-Nornuciferine \\
\hline 2-Hydroxy-1-methoxyaporphine & $\mathrm{OH}$ & $\mathrm{OCH}_{3}$ & $\mathrm{CH}_{3}$ & 2-Hydroxy-1-methoxyaporphine \\
\hline Roemerine & \multirow{2}{*}{\multicolumn{2}{|c|}{$\begin{array}{l}-\mathrm{O}-\mathrm{CH}_{2}-\mathrm{O}- \\
-\mathrm{O}-\mathrm{CH}_{2}-\mathrm{O}-\end{array}$}} & $\mathrm{CH}_{3}$ & Roemerine \\
\hline Anonaine & & & $\mathrm{H}$ & Anonaine \\
\hline
\end{tabular}<smiles>[R]c1cc2c3c(cc4ccccc4c3c1[R])N([R])CC2</smiles>

Fig. 4. Nucleus structures of dehydrogenase aporphin

\begin{tabular}{|c|c|c|c|}
\hline \multicolumn{4}{|c|}{$\begin{array}{c}\text { TABLE-3 } \\
\text { STRUCTURES OF SUBSTITUENT GROUPS } \\
\text { IN DEHYDROGENASE APORPHIN }\end{array}$} \\
\hline Compound & $\mathrm{R}_{2}$ & $\mathrm{R}_{3}$ & Name \\
\hline $\begin{array}{l}\text { Dehydronuciferinene } \\
\text { Dehydroroemerine }\end{array}$ & $\begin{array}{c}\mathrm{OCH}_{3} \quad \mathrm{OCH}_{3} \\
-\mathrm{O}-\mathrm{CH}_{2}-\mathrm{O}-\end{array}$ & $\begin{array}{l}\mathrm{CH}_{3} \\
\mathrm{CH}_{3}\end{array}$ & $\begin{array}{l}\text { Dehydronuciferinene } \\
\text { Dehydroroemerine }\end{array}$ \\
\hline
\end{tabular}

Aporphine alkaloids belong to isoquinoline type alkaloid, including pronuciferine, nuciferine, $\mathrm{N}$-nornuciferine, $\mathrm{O}$ nornuciferine, liriodenine, roemerine, $\mathrm{N}$-norarmepavine, 2hydroxy-1-methoxyaporphine.

Many researches showed that the alkaloids from Nelumbo nucifera had lipid-lowering, weight-losing, antibacterial and antiviral functions. However, review and systemic analysis of distribution, structures and pharmacological activities of aporphine alkaloids have not been reported. The author prompted to provide the currently available information on traditional and local knowledge, ethno biological and ethno medicinal issues and pharmacological studies on these useful plants. The aim of the present review is to introduce aporphine alkaloids as medicinal drugs by highlighting its traditional applications as well as the recent findings for novel pharmacological and clinical applications.

\section{Distribution of aporphine alkaloids in plants}

Aporphine alkaloids belong to isoquinoline type alkaloid. The basic structure of which is shown in Fig. 5(A). There are substituent groups at the positions of $\mathrm{C}_{1}, \mathrm{C}_{2}$ in the native aporphine alkaloids simultaneously and at the same time, substitution reactions may take place at the positions of $\mathrm{C}_{3,8,9,10,11}$.

Substituent groups mainly include $-\mathrm{OH},-\mathrm{OCH}_{3}, \mathrm{CH}_{2} \mathrm{Z}_{\mathrm{O}-}^{\mathrm{O}-}$ The substituent groups at the position of nitrogen atom are $-\mathrm{H},-\mathrm{CH}_{3},-\mathrm{COOCH}_{3}$, etc. This type of alkaloid mainly includes: alkaloid prototype (Fig. 5A), oxidized aporphine (Fig. 5B) and dehydro-aporphine (Fig. 5C).

Aporphine alkaloids broadly exist in nature and have obvious biological activity, with great content. Now hundreds of aporphine alkaloids have been isolated from more than 20 families and 100 genuses of plants.

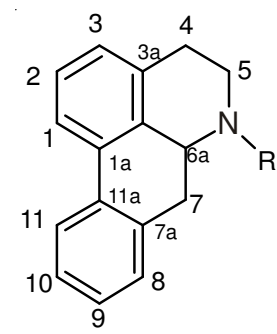

(A)

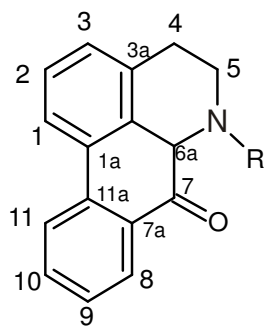

(B)<smiles>PN1CCc2cccc3c2c1cc1ccccc13</smiles>

(C)
Fig. 5. Three basic structures of aporphine alkaloids

\section{Distribution of aporphine alkaloids ${ }^{1-61}$}

Annonaceae: Annonaceae, with original traits, belongs to Magnoliopsida and this so-called "living fossil" is born in American tropics, broadly distributing in tropics and subtropics. Data shows that aporphine alkaloids have the widest distribution in Annonaceae. Aporphine alkaloids have been isolated from 28 genuses of these plants viz., Alphonsea, Anaxagorea, Annona, Artabotrys, Asimina, Cananga, Cheistophlis, Desmos, Duguetia, Enantia, Eupomatia, Fusea, Goniothalamus, Greenwayodendron, Guatteria, Hexalobus, Isolona, Meiocarpidium, Melodorum, Mitrella, Monanthotaxis, Monodora, Pachypodanthium, Polyalthia, Popowia, Pseuduvaria, Schefferomitra, Uvariopsis, Xylopia and Fissistigma.

Menispermaceae: There are about 70 genuses and more than 430 species of plants in Menispermaceae, which mainly distribute in tropics and subtropics. There are about 20 genuses and 60 species in China. Aporphine alkaloids have been isolated from 20 genuses of these plants e.g., Abuta, Anamirta, Cbasmanthera, Cissampelos, Cocculus, Coscinium, Cyclea, Dioscoreophyllum, Fibraurea, Heptacyclum, Kolobopetalum, Legnephora, Meiocarpidium, Menispermum, Pachygone, Pycnarrhena, Rbigiocarya, Sinomenium, Stephania, Telitoxicum, Tiliacora, Tinospora and Triclisia.

Papaveraceae: There are about 23 genuses in Papaveraceae, which broadly distribute in temperate zones and subtropics and most of them are herbs. Aporphine alkaloids have been isolated from 13 genuses of these plants e.g., Argemone, Chelidonium, Corydalis, Dicentra, Dicranostigma, Eschscholtzia, Fumaria, Glaucium, Meconopsis, Papaver, Pteridophyllum, Roemeria and Platycapnos.

Ranunculaceae: Annonaceae, with 50 genuses and more than 2000 species, belongs to Saxifragales, which broadly distributes around the whole world, especially in the north temperate zone and frigid zone and there are 39 genuses and about 750 species of these plants in China. Aporphine alkaloids have been isolated from 9 genuses of these plants i.e., Aconitum, Aquilegia, Caltha, Coptis, Delphinium, Isopyrum, Nigella, Thalictrum and Trollius. 
Lauraceae: There are 45 genuses and more than 2000 species in Lauracea, mainly distributing in tropics and subtropic. There are 20 genuses and more than 420 species in China, mainly distributing in areas south of the Qinling mountain/Huaihe river. Aporphine alkaloids have been isolated from 18 genuses of these plants i.e., Actinodaphne, Alseodaphne, Beilschmiedia, Cassytha, Cinnamomum, Cryptocarya, Dehaasia, Laurus, Lindera, Litsea, Machilus, Mezilaurus, Nectandra, Neolitsea, Ocotea, Phoebe, Ravensara and Sassafras.

Monimiceae: There are about 18-25 genuses and 150220 species in Monimiceae, distributing in the Southern Hemisphere and some of them are shrubs and dungarungas. Aporphine alkaloids have been isolated from 10 genuses of these plants i.e., Atherosperma, Doryphora, Dryadodaphne, Laurelia, Laureliopsis, Monimia, Nemuaron, Palmeria, Peumus and Siparuna.

Magnoliaceae: Magnoliaceae, with original flowers, belongs to dicotyledon and there are 7 genuses and 225 species in it, mainly distributing in tropics and subtropics of Asia, especially in southern China and Indochina, with a few species in America. Aporphine alkaloids have been isolated from 6 genuses of these plants i.e., Elmerrillia, Liriodendron, Magnolia, Michelia, Talauma and Tsoongiodendron.

Berberidaceae: There are 15 genuses and 570 species in Berberidaceae and most of them belong to Berberis. Aporphine alkaloids have been isolated from 4 genuses of these plants i.e., Berberis, Leonitice, Machonia and Nandina.

Aristolochiaceae: Aristolochiaceae, with 7 genuses and 350 species, belongs to dicotyledon, broadly distributing in tropics and temperate zones, especially in southern America. Aporphine alkaloids have been isolated from 2 genuses of these plants i.e., Aristolochia and Bragantia.

Euphorbiaceae: Euphorbiaceae, with 300 genuses and more than 8000 species, belongs to dicotyledon, broadly distributing around the world. Aporphine alkaloids have been isolated from 2 genuses of these plants i.e., Caullophyllum and Croton.

Hernandiaceae: Hernandiaceae, with 3 genuses and 54 species, belongs to dicotyledon, mainly distributing in tropics. Aporphine alkaloids have been isolated from 2 genuses of these plants i.e., Hernandia and Illigera.

Rataceae: Rataceae, with 150 genuses and about 900 species, belongs to dicotyledon, distributing in tropics and temperate zones, mostly in South Africa and Oceania. Aporphine alkaloids have been isolated from 2 genuses of these plants i.e.,Fagara and Phellodendron.

Rhamnaceae: Rhamnaceae, with 58 genuses and about 900 species, belongs to dicotyledon, broadly distributing around the world. Aporphine alkaloids have been isolated from 4 genuses of these plants i.e., Colubrina, Ziziphus, Phylica and Retanilla.

Araceae: Araceae, with 115 genuses and more than 2000 species, belongs to monocotyledon, broadly distributing around the world and more than $92 \%$ of them are in tropics. Aporphine alkaloids have been isolated from Lysichiton.

Canellaceae: Canellaceae, with 6 genuses, grow in tropics, mainly distributing in South America, West Indies,
East Africa and Madagascar. Aporphine alkaloids have been isolated from Cinnamosma.

Leguminoseae: Leguminoseae, with 690 genuses, is the third largest family of spermatophyte, broadly distributing around the world. Aporphine alkaloids have been isolated from 2 genuses of these plants i.e., Erythrina and Euchresta.

Liliaceae: Liliaceae, with 175 genuses and more than 2000 species, broadly distributes around the world and specially in temperate zones and subtropics. Aporphine alkaloids have been isolated from Baeometra.

Nymphaeaceae: Nymphaeaceae, with 8 genuses and about 100 species, belongs to dicotyledon, mainly distributes in temperate zones and tropics. Aporphine alkaloids have been isolated from Nelumbo.

Piperaceae: Piperaceae, with 8 or 9 genuses and about 3100 species, belongs to dicotyledon, mainly distributing in tropics and subtropics. Aporphine alkaloids have been isolated from Piper.

Symplocaceae: Symplocaceae, with 250 species, mainly distributes in tropics and subtropics. Aporphine alkaloids have been isolated from Symplocos.

Sabiaceae: Sabiaceae, with 3 genuses and about 150 species, belongs to dicotyledon, mainly distributing between tropics and temperate zones. Aporphine alkaloids have been isolated from Sabia.

Fumariaceae: Corydalis.

\section{Structures of aporphine alkaloids}

The basic structure of aporphine alkaloids is shown in Fig. 6. The names of aporphine compounds, types of substituent groups and botanical sources are shown in Table- 4 .

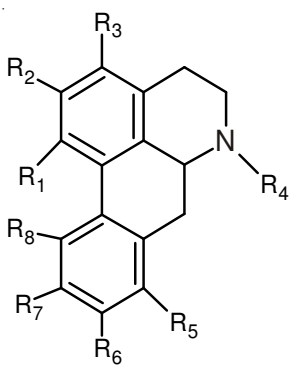

Fig. 6. Basic structure of aporphine alkaloids

\section{Pharmacological activity of aporphine alkaloids}

Pharmacological activity on adrenoreceptor: Pharmacological activity on blocking adrenoreceptor is one of the widest and most important bioactivities of aporphine alkaloids, especially in $\alpha$-adrenoceptor, such as $\mathrm{N}$-methylactinodaphnine $^{182},(+/-)$-domesticine ${ }^{181}$, corytuberine ${ }^{92}$, magnoflorine ${ }^{92}$, isothebaine ${ }^{92}$, isocorydine ${ }^{92}$, Dicentrine ${ }^{183}$, S-(+)-boldine ${ }^{184}$.

Firstly, most of these alkaloids have effect on blocking $\alpha_{1}$-adrenoceptor, such as N-methyl-actinodaphnine ${ }^{189},(+/-)$ domesticine $^{181}$, dicentrine ${ }^{183}$, while a few aporphine alkaloids, such as (+)-nantenine ${ }^{185}$, have the effect of antagonism in $\alpha_{1^{-}}$ adrenoceptor and $\alpha_{2}$-adrenoceptor simultaneously. Different aporphine alkaloids have selectivity for different subtypes of receptor and $\mathrm{N}$-methyl-actinodaphnine ${ }^{189}$ has more affinity 


\begin{tabular}{|c|c|c|c|c|c|c|c|c|c|}
\hline \multirow{3}{*}{ Names of compounds } & \multicolumn{8}{|c|}{$\begin{array}{c}\text { TABLE-4 } \\
\text { APORPHINE ALKALOIDS }\end{array}$} & \multirow{3}{*}{ Botanical sources } \\
\hline & \multicolumn{8}{|c|}{ Structure of compound } & \\
\hline & $\mathrm{R}_{1}$ & $\mathrm{R}_{2}$ & $\mathrm{R}_{3}$ & $\mathrm{R}_{4}$ & $\mathrm{R}_{5}$ & $\mathrm{R}_{6}$ & $\mathrm{R}_{7}$ & $\mathrm{R}_{8}$ & \\
\hline Caaverine & $-\mathrm{OH}$ & $-\mathrm{OCH}_{3}$ & $-\mathrm{H}$ & $-\mathrm{H}$ & $-\mathrm{H}$ & $-\mathrm{H}$ & $-\mathrm{H}$ & $-\mathrm{H}$ & $\begin{array}{l}\text { Ocotea lancifolia }{ }^{[62]} \\
\text { Celastrinea } \text { Mart }^{[180]}\end{array}$ \\
\hline Lirinidine & $-\mathrm{OH}$ & $-\mathrm{OCH}_{3}$ & $-\mathrm{H}$ & $-\mathrm{CH}_{3}$ & $-\mathrm{H}$ & $-\mathrm{H}$ & $-\mathrm{H}$ & $-\mathrm{H}$ & $\begin{array}{l}\text { Annona purpurea }{ }^{[207]} \\
\text { Nelumbo nucifera }^{[64]} \\
\text { Papaver armeniacum }{ }^{[65]} \\
\text { Artabotrys venustus }{ }^{[67]} \\
\text { Magnolia officinalis } \text { Rehder et W ils }{ }^{[66]}\end{array}$ \\
\hline Asimilobine & $-\mathrm{OCH}_{3}$ & $-\mathrm{OH}$ & $-\mathrm{H}$ & $-\mathrm{H}$ & $-\mathrm{H}$ & $-\mathrm{H}$ & $-\mathrm{H}$ & $-\mathrm{H}$ & 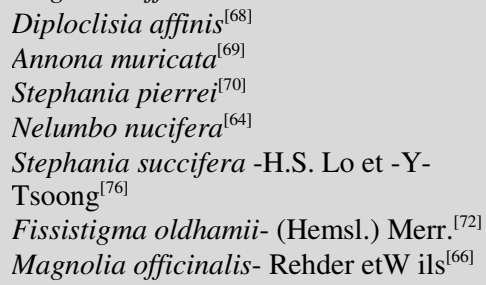 \\
\hline N-Methyl-asimilobine & $-\mathrm{OCH}_{3}$ & $-\mathrm{OH}$ & $-\mathrm{H}$ & $-\mathrm{CH}_{3}$ & $-\mathrm{H}$ & $-\mathrm{H}$ & $-\mathrm{H}$ & $-\mathrm{H}$ & $\begin{array}{l}\text { Annona purpurea }{ }^{[125]} \\
\text { Papaver cylindricum }^{[73]} \\
\text { Egyptian Papaver rhoea }^{[74]} \\
\text { Nelumbo lutea (Wild.) pers. }{ }^{[75]}\end{array}$ \\
\hline Nornuciferine & $-\mathrm{OCH}_{3}$ & $-\mathrm{OCH}_{3}$ & $-\mathrm{H}$ & $-\mathrm{H}$ & $-\mathrm{H}$ & $-\mathrm{H}$ & $-\mathrm{H}$ & $-\mathrm{H}$ & $\begin{array}{l}\text { Guatteria spp. }{ }^{[76]} \\
\text { Annona muricata }{ }^{[69]} \\
\text { Chasmanthera dependens } s^{[77]} \\
\text { Nelumbo lutea (Wild.) -pers. }{ }^{[75]}\end{array}$ \\
\hline Nuciferine & $-\mathrm{OCH}_{3}$ & $-\mathrm{OCH}_{3}$ & $-\mathrm{H}$ & $-\mathrm{CH}_{3}$ & $-\mathrm{H}$ & $-\mathrm{H}$ & $-\mathrm{H}$ & $-\mathrm{H}$ & $\begin{array}{l}\text { Nelumbinis }{ }^{[78]} \\
\text { Papaver armeniacum }^{[94]} \\
\text { P. } \text { fugax }^{[94]} \\
\text { P. } \text { tauricola }^{[94]} \\
\text { Nelumbo lutea (Wild.) -pers }^{[75]}\end{array}$ \\
\hline Anonaine & $-\mathrm{O}-\mathrm{C}$ & $\mathrm{H}_{2}-\mathrm{O}-$ & $-\mathrm{H}$ & $-\mathrm{H}$ & $-\mathrm{H}$ & $-\mathrm{H}$ & $-\mathrm{H}$ & $-\mathrm{H}$ & $\begin{array}{l}\text { Annona salzmanii D.C. }{ }^{[79]} \\
\text { Annona squamosa }{ }^{[80]} \\
\text { Nelumbo lutea }\left(\text { Wild.) -pers. }{ }^{[77]}\right. \\
\text { D. tiebaghiensis }{ }^{[225]} \\
\text { Magnolia officinalis- Rehder etW ils }{ }^{[82]}\end{array}$ \\
\hline N-Methylanonaine ${ }^{[1]}$ & $-\mathrm{O}-\mathrm{C}$ & $\mathrm{H}_{2}-\mathrm{O}-$ & $-\mathrm{H}$ & $-\mathrm{CH}_{3}$ & $-\mathrm{H}$ & $-\mathrm{H}$ & $-\mathrm{H}$ & $-\mathrm{H}$ & $\begin{array}{l}\text { Annonaceae: Annona, Xylopia } \\
\text { Lauraceae: Cryptocarya, Neolitsea } \\
\text { Magnoliaceae: Liriodendron } \\
\text { Menispermaceae: Stephania } \\
\text { Monimiaceae: Laurelia } \\
\text { Nympheaceae: Nelumbo } \\
\text { Papaverceae: Papaver, Roemeria. } \\
\text { Rhamnaceae: Colubrina }\end{array}$ \\
\hline Roemrefidine & $-\mathrm{O}-\mathrm{C}$ & $\mathrm{H}_{2}-\mathrm{O}-$ & $-\mathrm{H}$ & $\left(-\mathrm{CH}_{3}\right)_{2}$ & $-\mathrm{H}$ & $-\mathrm{H}$ & $-\mathrm{H}$ & $-\mathrm{H}$ & Sparattanthelium amazonum ${ }^{[83]}$ \\
\hline Stephalagine & $-\mathrm{O}-\mathrm{C}$ & $\mathrm{H}_{2}-\mathrm{O}-$ & $-\mathrm{OCH}_{3}$ & $-\mathrm{CH}_{3}$ & $-\mathrm{H}$ & $-\mathrm{H}$ & $-\mathrm{H}$ & $-\mathrm{H}$ & Stephania dinklagei ${ }^{[84]}$ \\
\hline Stephanine & $-\mathrm{O}-\mathrm{C}$ & $\mathrm{H}_{2}-\mathrm{O}-$ & $-\mathrm{H}$ & $-\mathrm{CH}_{3}$ & $-\mathrm{OCH}_{3}$ & $-\mathrm{H}$ & $-\mathrm{H}$ & $-\mathrm{H}$ & $\begin{array}{l}\text { Stephania bancroftii F.M. -Bailey }{ }^{[85]} \\
\text { S. aculeate F.M. Bailey }\end{array}$ \\
\hline Lirinine & $-\mathrm{OH}$ & $-\mathrm{OCH}_{3}$ & $-\mathrm{H}$ & $-\mathrm{CH}_{3}$ & $-\mathrm{H}$ & $-\mathrm{OCH}_{3}$ & $-\mathrm{H}$ & $-\mathrm{H}$ & Artabotrys hainanensi ${ }^{[149]}$ \\
\hline $\begin{array}{l}\text { Lirinine } \mathrm{N} \text {-oxide }{ }^{[1]} \\
(\mathrm{N} \rightarrow \mathrm{O})\end{array}$ & $-\mathrm{OH}$ & $-\mathrm{OCH}_{3}$ & $-\mathrm{H}$ & $-\mathrm{CH}_{3}$ & $-\mathrm{H}$ & $-\mathrm{OCH}_{3}$ & $-\mathrm{H}$ & $-\mathrm{H}$ & Magnoliaceae: Liriodendron \\
\hline O-methyllirinine ${ }^{[1]}$ & $-\mathrm{OCH}_{3}$ & $-\mathrm{OCH}_{3}$ & $-\mathrm{H}$ & $-\mathrm{CH}_{3}$ & $-\mathrm{H}$ & $-\mathrm{OCH}_{3}$ & $-\mathrm{H}$ & $-\mathrm{H}$ & Magnoliaceae: Liriodendron \\
\hline Anolobine & $-\mathrm{O}-\mathrm{C}$ & $\mathrm{H}_{2}-\mathrm{O}-$ & $-\mathrm{H}$ & $-\mathrm{H}$ & $-\mathrm{H}$ & $-\mathrm{OH}$ & $-\mathrm{H}$ & $-\mathrm{H}$ & Magnolia coco (Lour.)DC. ${ }^{[86]}$ \\
\hline Roemerine & $-\mathrm{O}-\mathrm{C}$ & $\mathrm{H}_{2}-\mathrm{O}-$ & $-\mathrm{H}$ & $-\mathrm{CH}_{3}$ & $-\mathrm{H}$ & $-\mathrm{OH}$ & $-\mathrm{H}$ & $-\mathrm{H}$ & $\begin{array}{l}\text { Hornschuchia oblique } e^{[87]} \\
\text { Annona cherimolia }^{[88]} \\
\text { Annona senegalensis }^{[89]} \\
\text { Papaver armeniacum } \\
\text { Nelumbo lutea } \text { (Wild.) pers. }{ }^{[75]} \\
\text { Stephania kwangsiensis } \text {-H.S. } \text { Lo }^{[17]}\end{array}$ \\
\hline O-Methylanolobine ${ }^{[1]}$ & $-\mathrm{O}-\mathrm{C}$ & $\mathrm{H}_{2}-\mathrm{O}-$ & $-\mathrm{H}$ & $-\mathrm{H}$ & $-\mathrm{H}$ & $-\mathrm{OCH}_{3}$ & $-\mathrm{H}$ & $-\mathrm{H}$ & Annonaceae: Xylopia \\
\hline Isolaureline & $-\mathrm{O}-\mathrm{C}$ & $\mathrm{H}_{2}-\mathrm{O}-$ & $-\mathrm{H}$ & $-\mathrm{CH}_{3}$ & $-\mathrm{H}$ & $-\mathrm{OCH}_{3}$ & $-\mathrm{H}$ & $-\mathrm{H}$ & Fissistigma oldhamii-(Hemsl.)Merr. ${ }^{[72]}$ \\
\hline Sparsiflorine $\mathrm{e}^{[1]}$ & $-\mathrm{OH}$ & $-\mathrm{OCH}_{3}$ & $-\mathrm{H}$ & $-\mathrm{H}$ & $-\mathrm{H}$ & $-\mathrm{H}$ & $-\mathrm{OH}$ & $-\mathrm{H}$ & Croton sparsiflorus-Morung \\
\hline Apoglaziovine & $-\mathrm{OH}$ & $-\mathrm{OCH}_{3}$ & $-\mathrm{H}$ & $-\mathrm{CH}_{3}$ & $-\mathrm{H}$ & $-\mathrm{H}$ & $-\mathrm{OH}$ & $-\mathrm{H}$ & Berberis brandisiana ${ }^{[90]}$ \\
\hline Variabiline $^{[1]}$ & $-\mathrm{OH}$ & $-\mathrm{OCH}_{3}$ & $-\mathrm{H}$ & $-\mathrm{CH}_{3}$ & $-\mathrm{H}$ & $-\mathrm{H}$ & $\begin{array}{l}-\mathrm{N}\left(\mathrm{CH}_{2-}^{-}\right. \\
\left.\mathrm{C}_{6} \mathrm{H}_{6}\right)_{2}\end{array}$ & $-\mathrm{H}$ & Lauraceae: Coctea \\
\hline Apocrotonosine $^{[1]}$ & $-\mathrm{OCH}_{3}$ & $-\mathrm{OH}$ & $-\mathrm{H}$ & $-\mathrm{H}$ & $-\mathrm{H}$ & $-\mathrm{H}$ & $-\mathrm{OH}$ & $-\mathrm{H}$ & Euphorbiaceae: Croton \\
\hline
\end{tabular}




\begin{tabular}{|c|c|c|c|c|c|c|c|c|c|}
\hline Phoebe base II $^{[1]}$ & $-\mathrm{OCH}_{3}$ & $-\mathrm{OH}$ & $-\mathrm{H}$ & $-\mathrm{CH}_{3}$ & $-\mathrm{H}$ & $-\mathrm{OCH}_{3}$ & $-\mathrm{H}$ & $-\mathrm{H}$ & Lauraceae: Phoebe \\
\hline Tuduranine & $-\mathrm{OCH}_{3}$ & $-\mathrm{OCH}_{3}$ & $-\mathrm{H}$ & $-\mathrm{H}$ & $-\mathrm{H}$ & $-\mathrm{H}$ & $-\mathrm{OH}$ & $-\mathrm{H}$ & S. rotunda, $S$. sasakii ${ }^{[91]}$ \\
\hline Nuciferoline $^{[1]}$ & $-\mathrm{OCH}_{3}$ & $-\mathrm{OCH}_{3}$ & $-\mathrm{H}$ & $-\mathrm{CH}_{3}$ & $-\mathrm{H}$ & $-\mathrm{H}$ & $-\mathrm{OH}$ & $-\mathrm{H}$ & Papaverceae: Papaver \\
\hline Mecambroline $^{[1]}$ & \multicolumn{2}{|c|}{$-\mathrm{O}-\mathrm{CH}_{2}-\mathrm{O}-$} & $-\mathrm{H}$ & $-\mathrm{CH}_{3}$ & $-\mathrm{H}$ & $-\mathrm{H}$ & $-\mathrm{OH}$ & $-\mathrm{H}$ & $\begin{array}{l}\text { Lauraceae: Phoebe } \\
\text { Monimiaceae: Laurelia } \\
\text { Meconopsis } \\
\text { Papaverceae: Meconopsis, Papaver }\end{array}$ \\
\hline Michiepressine ${ }^{[195]}$ & \multicolumn{2}{|c|}{$-\mathrm{O}-\mathrm{CH}_{2}-\mathrm{O}-$} & $-\mathrm{H}$ & $\left(-\mathrm{CH}_{3}\right)_{2}$ & $-\mathrm{H}$ & $-\mathrm{H}$ & $-\mathrm{OH}$ & $-\mathrm{H}$ & Magnoliaceae: Michelia \\
\hline Laureline & \multicolumn{2}{|c|}{$-\mathrm{O}-\mathrm{CH}_{2}-\mathrm{O}-$} & $-\mathrm{H}$ & $-\mathrm{CH}_{3}$ & $-\mathrm{H}$ & $-\mathrm{H}$ & $-\mathrm{OCH}_{3}$ & $-\mathrm{H}$ & Guatteria elata $^{[98]}$ \\
\hline Isothebaine & $-\mathrm{OH}$ & $-\mathrm{OCH}_{3}$ & $-\mathrm{H}$ & $-\mathrm{CH}_{3}$ & $-\mathrm{H}$ & $-\mathrm{H}$ & $-\mathrm{H}$ & $-\mathrm{OCH}_{3}$ & $\begin{array}{l}\text { Mahonia aquifolium }{ }^{[99]} \\
\text { Papaver bracteatum } \\
\text { Papaver orientale }^{[101]} \\
\text { P. pseudo-orientale } \\
{[101]}\end{array}$ \\
\hline Obovanine $^{[1]}$ & \multicolumn{2}{|c|}{$-\mathrm{O}-\mathrm{CH}_{2}-\mathrm{O}-$} & $-\mathrm{H}$ & $-\mathrm{H}$ & $-\mathrm{H}$ & $-\mathrm{H}$ & $-\mathrm{H}$ & $-\mathrm{OH}$ & Magnoliaceae: Magnolia \\
\hline Pukateine $^{[1]}$ & \multicolumn{2}{|c|}{$-\mathrm{O}-\mathrm{CH}_{2}-\mathrm{O}-$} & $-\mathrm{H}$ & $-\mathrm{CH}_{3}$ & $-\mathrm{H}$ & $-\mathrm{H}$ & $-\mathrm{H}$ & $-\mathrm{OH}$ & Guatteria elata \\
\hline Laurepukine $^{[1]}\left(\mathrm{N}^{+}-\mathrm{O}^{-}\right)$ & \multicolumn{2}{|c|}{$-\mathrm{O}-\mathrm{CH}_{2}-\mathrm{O}-$} & $-\mathrm{H}$ & $-\mathrm{CH}_{3}$ & $-\mathrm{H}$ & $-\mathrm{H}$ & $-\mathrm{H}$ & $-\mathrm{OH}$ & Monimiaceae: Laurelia \\
\hline O-Methylpukateine ${ }^{[1]}$ & \multicolumn{2}{|c|}{$-\mathrm{O}-\mathrm{CH}_{2}-\mathrm{O}-$} & $-\mathrm{H}$ & $-\mathrm{CH}_{3}$ & $-\mathrm{H}$ & $-\mathrm{H}$ & $-\mathrm{H}$ & $-\mathrm{OCH}_{3}$ & Monimiaceae: Laurelia \\
\hline Crebaine & \multicolumn{2}{|c|}{$-\mathrm{O}-\mathrm{CH}_{2}-\mathrm{O}-$} & $-\mathrm{H}$ & $-\mathrm{CH}_{3}$ & $-\mathrm{OCH}_{3}$ & $-\mathrm{OCH}_{3}$ & $-\mathrm{H}$ & $-\mathrm{H}$ & $\begin{array}{l}\text { S. cepharantha } a^{95]} \\
\text { S. dielaiana } a^{[95]} \\
\text { S. venosa }{ }^{[95]}\end{array}$ \\
\hline Laurelliptine & $-\mathrm{OH}$ & $-\mathrm{OCH}_{3}$ & $-\mathrm{H}$ & $-\mathrm{H}$ & $-\mathrm{H}$ & $-\mathrm{OH}$ & $-\mathrm{OCH}_{3}$ & $-\mathrm{H}$ & $\begin{array}{l}\text { Annona salzmanii D.C. }{ }^{[238]} \\
\text { Nectandra rigida }{ }^{[96]}\end{array}$ \\
\hline Isoboldine & $-\mathrm{OH}$ & $-\mathrm{OCH}_{3}$ & $-\mathrm{H}$ & $-\mathrm{CH}_{3}$ & $-\mathrm{H}$ & $-\mathrm{OH}$ & $-\mathrm{OCH}_{3}$ & $-\mathrm{H}$ & 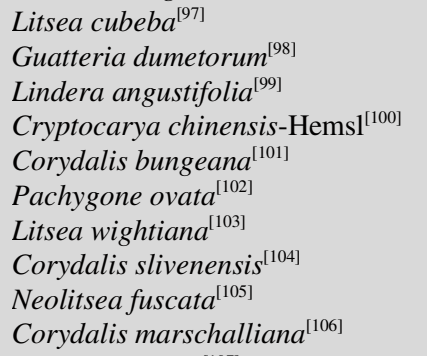 \\
\hline Laurifoline & $-\mathrm{OH}$ & $-\mathrm{OCH}_{3}$ & $-\mathrm{H}$ & $\left(-\mathrm{CH}_{3}\right)_{2}$ & $-\mathrm{H}$ & $-\mathrm{OH}$ & $-\mathrm{OCH}_{3}$ & $-\mathrm{H}$ & Hypserpa nitida ${ }^{[107]}$ \\
\hline Bracteoline $^{[1]}$ & $-\mathrm{OH}$ & $-\mathrm{OCH}_{3}$ & $-\mathrm{H}$ & $-\mathrm{CH}_{3}$ & $-\mathrm{H}$ & $-\mathrm{OCH}_{3}$ & $-\mathrm{OH}$ & $-\mathrm{H}$ & Papaverceae: Papaver \\
\hline Wilsonirine & $-\mathrm{OH}$ & $-\mathrm{OCH}_{3}$ & $-\mathrm{H}$ & $-\mathrm{H}$ & $-\mathrm{H}$ & $-\mathrm{OCH}_{3}$ & $-\mathrm{OCH}_{3}$ & $-\mathrm{H}$ & A. monteiroae $e^{[108]}$ \\
\hline Thaliporphine & $-\mathrm{OH}$ & $-\mathrm{OCH}_{3}$ & $-\mathrm{H}$ & $-\mathrm{CH}_{3}$ & $-\mathrm{H}$ & $-\mathrm{OCH}_{3}$ & $-\mathrm{OCH}_{3}$ & $-\mathrm{H}$ & $\begin{array}{l}\text { Croton lechleri }^{[109]} \\
\text { Neolitsea konishii } \mathrm{K}^{[110]} \\
\text { Corydalis bulbosa }^{[111]} \\
\text { Mahonia repens }{ }^{[5]} \\
\text { Corydalis yanhusuo }^{[112]}\end{array}$ \\
\hline Thalicmidine $^{[1]}(\mathrm{N} \rightarrow \mathrm{O})$ & $-\mathrm{OH}$ & $-\mathrm{OCH}_{3}$ & $-\mathrm{H}$ & $-\mathrm{CH}_{3}$ & $-\mathrm{H}$ & $-\mathrm{OCH}_{3}$ & $-\mathrm{OCH}_{3}$ & $-\mathrm{H}$ & Ranuncudaceae: Thalictrum \\
\hline Fagara base $^{[1]}$ & $-\mathrm{OH}$ & $-\mathrm{OCH}_{3}$ & $-\mathrm{H}$ & $\left(-\mathrm{CH}_{3}\right)_{2}$ & $-\mathrm{H}$ & $-\mathrm{OCH}_{3}$ & $-\mathrm{OCH}_{3}$ & $-\mathrm{H}$ & Rutaceae: Fagara \\
\hline Nordomesticine & $-\mathrm{OH}$ & $-\mathrm{OCH}_{3}$ & $-\mathrm{H}$ & $-\mathrm{H}$ & $-\mathrm{H}$ & $-\mathrm{O}-\mathrm{Cl}$ & $\mathrm{I}_{2}-\mathrm{O}-$ & $-\mathrm{H}$ & $\begin{array}{l}\text { Nandina domestica }{ }^{[113]} \\
\text { Corydalis slivenensis }^{[104]}\end{array}$ \\
\hline Domesticine & $-\mathrm{OH}$ & $-\mathrm{OCH}_{3}$ & $-\mathrm{H}$ & $-\mathrm{CH}_{3}$ & $-\mathrm{H}$ & $-\mathrm{O}-\mathrm{Cl}$ & $\mathrm{I}_{2}-\mathrm{O}-$ & $-\mathrm{H}$ & $\begin{array}{l}\text { Nandina domestica }{ }^{[113]} \\
\text { Corydalis slivenensis }{ }^{[104]} \\
\text { C. susaveolens Hance }{ }^{[114]}\end{array}$ \\
\hline Laurolistsine & $-\mathrm{OCH}_{3}$ & $-\mathrm{OH}$ & $-\mathrm{H}$ & $-\mathrm{H}$ & $-\mathrm{H}$ & $-\mathrm{OH}$ & $-\mathrm{OCH}_{3}$ & $-\mathrm{H}$ & $\begin{array}{l}\text { Litsea rotundifolia var. - } \\
\text { oblongifolia }^{[115]}\end{array}$ \\
\hline Boldine & $-\mathrm{OCH}_{3}$ & $-\mathrm{OH}$ & $-\mathrm{H}$ & $-\mathrm{CH}_{3}$ & $-\mathrm{H}$ & $-\mathrm{OH}$ & $-\mathrm{OCH}_{3}$ & $-\mathrm{H}$ & $\begin{array}{l}\text { Phoebe grandis }{ }^{[116]} \\
\text { Lindera aggregate }^{[117]} \\
\text { Litsea sessilis }^{[118]} \\
\text { Lindera angustifolia }^{[46]} \\
\text { Cassytha filiformis }{ }^{[119]} \\
\text { Peumus boldus } \text { Mol. }^{[120]} \\
\text { Litsea wightiana }^{[1103]} \\
\text { Lindera aggregate-Kosterm }^{[121]}\end{array}$ \\
\hline Norpredicentrine & $-\mathrm{OCH}_{3}$ & $-\mathrm{OH}$ & $-\mathrm{H}$ & $-\mathrm{H}$ & $-\mathrm{H}$ & $-\mathrm{OCH}_{3}$ & $-\mathrm{OCH}_{3}$ & $-\mathrm{H}$ & Lindera fragrans O-liv. ${ }^{[122]}$ \\
\hline Predicentrine & $-\mathrm{OCH}_{3}$ & $-\mathrm{OH}$ & $-\mathrm{H}$ & $-\mathrm{CH}_{3}$ & $-\mathrm{H}$ & $-\mathrm{OCH}_{3}$ & $-\mathrm{OCH}_{3}$ & $-\mathrm{H}$ & $\begin{array}{l}\text { Desmos rostrata }{ }^{[123]} \\
\text { Aromadendron elegans }^{[124]} \\
\text { Annona purpurea }{ }^{[258]} \\
\text { Cassytha filiformis } \mathrm{II}^{[125]} \\
\text { Corydalis slivenensis }^{[104]}\end{array}$ \\
\hline Isodomesticine & $-\mathrm{OCH}_{3}$ & $-\mathrm{OH}$ & $-\mathrm{H}$ & $-\mathrm{CH}_{3}$ & $-\mathrm{H}$ & $-\mathrm{O}-\mathrm{Cl}$ & & $-\mathrm{H}$ & Guatteria dumetorum $^{[98]}$ \\
\hline Laurotetanine & $-\mathrm{OCH}_{3}$ & $-\mathrm{OCH}_{3}$ & $-\mathrm{H}$ & $-\mathrm{H}$ & $-\mathrm{H}$ & $-\mathrm{OH}$ & $-\mathrm{OCH}_{3}$ & $-\mathrm{H}$ & 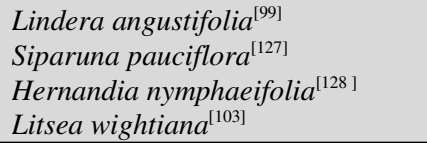 \\
\hline
\end{tabular}




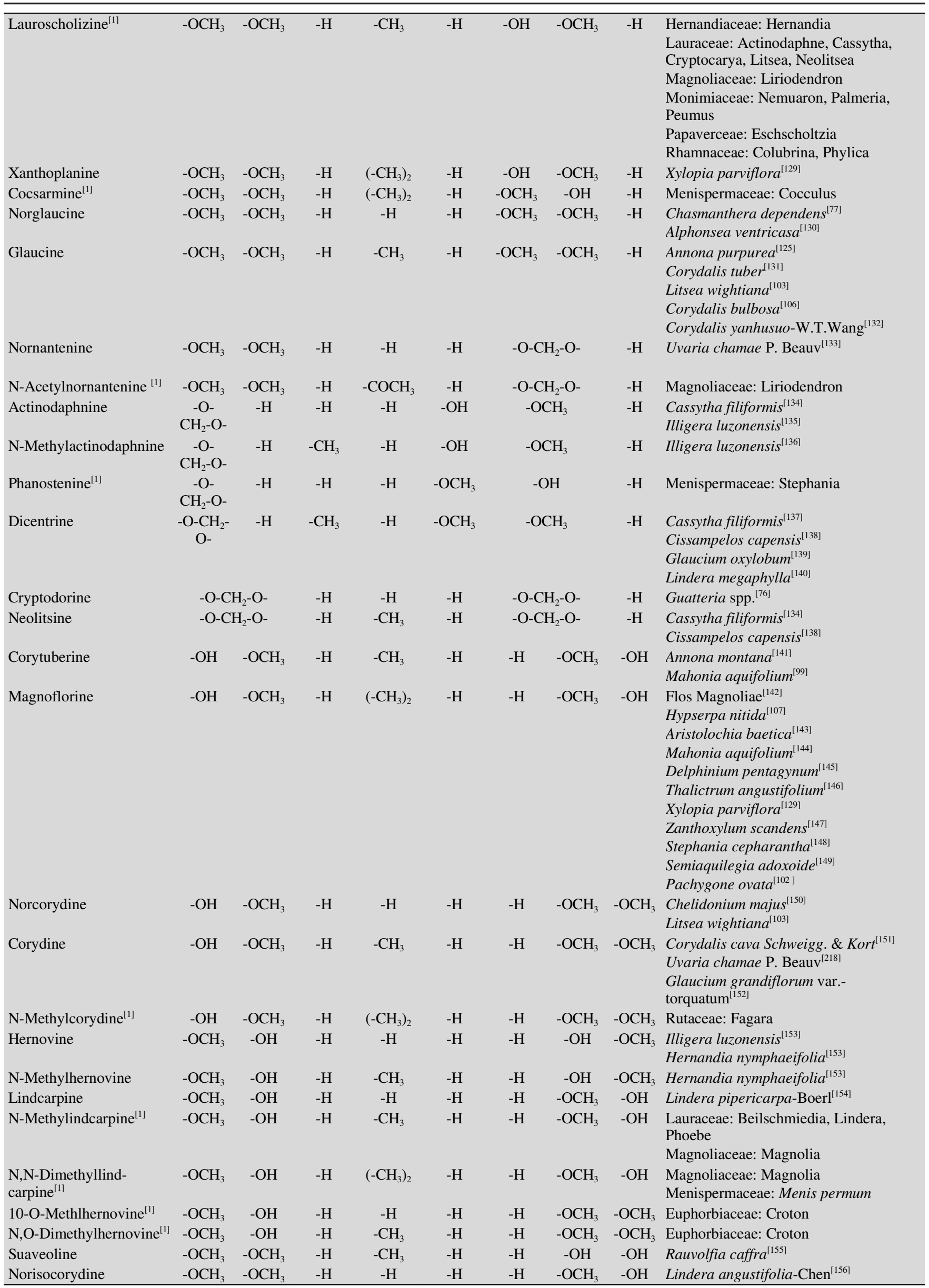




\begin{tabular}{|c|c|c|c|c|c|c|c|c|c|}
\hline Isocorydine & $-\mathrm{OCH}_{3}$ & $-\mathrm{OCH}_{3}$ & $-\mathrm{H}$ & $-\mathrm{CH}_{3}$ & $-\mathrm{H}$ & $-\mathrm{H}$ & $-\mathrm{OCH}_{3}$ & $-\mathrm{OH}$ & $\begin{array}{l}\text { Dactylicapnos scandens }{ }^{[157]} \\
\text { Stephania dinklagei }{ }^{[84]} \\
\text { Mahonia aquifolium }^{[92]} \\
\text { Thalictrum fauriei }^{[158]} \\
\text { Dactylicapnos torulosa }^{[159]} \\
\text { Glaucium grandiflorum var.- } \\
\text { torquatum }^{[152]} \\
\text { Corydalis slivenensis } \\
\text { Glaucium flavum-Crantz }^{[160]} \\
\text { Papaver armeniacum }^{[65]} \\
\text { Egyptian Papaver-rhoeas }^{[74]} \\
\text { Stephania kwangsiensis } \text { H. S.- Lo }^{[17]}\end{array}$ \\
\hline Menisperine & $-\mathrm{OCH}_{3}$ & $-\mathrm{OCH}_{3}$ & $-\mathrm{H}$ & $\left(-\mathrm{CH}_{3}\right)_{2}$ & $-\mathrm{H}$ & $-\mathrm{H}$ & $-\mathrm{OCH}_{3}$ & $-\mathrm{OH}$ & $\begin{array}{l}\text { Xylopia parviflora }{ }^{[129]} \\
\text { Stephania cepharantha }{ }^{[148]} \\
\text { Corydalis decumbens }-\left(\text { Thunb.) Pers. }{ }^{[161]}\right. \\
\text { Tinospora capillipes }^{[162]} \\
\text { Magnolia } \text { grandiflora }^{[163]}\end{array}$ \\
\hline Catalpifoline $\mathrm{C}^{[2]}$ & $-\mathrm{OCH}_{3}$ & $-\mathrm{OCH}_{3}$ & $-\mathrm{H}$ & $-\mathrm{H}$ & $-\mathrm{H}$ & $-\mathrm{H}$ & $-\mathrm{OCH}_{3}$ & $-\mathrm{OCH}_{3}$ & Hernandiaceae: Hernandia \\
\hline $\begin{array}{l}\text { O,O-Dimethycory- } \\
\text { tuberine } \mathrm{e}^{[2]}\end{array}$ & $-\mathrm{OCH}_{3}$ & $-\mathrm{OCH}_{3}$ & $-\mathrm{H}$ & $-\mathrm{CH}_{3}$ & $-\mathrm{H}$ & $-\mathrm{H}$ & $-\mathrm{OCH}_{3}$ & $-\mathrm{OCH}_{3}$ & Hernandiaceae: Hernandia \\
\hline Nandigerine $^{[2]}$ & $-\mathrm{O}-\mathrm{Cl}$ & $\mathrm{I}_{2}-\mathrm{O}-$ & $-\mathrm{H}$ & $-\mathrm{H}$ & $-\mathrm{H}$ & $-\mathrm{H}$ & $-\mathrm{OH}$ & $-\mathrm{OCH}_{3}$ & Hernandiaceae: Hernandia \\
\hline N-Methylhernangerine & $-\mathrm{O}-\mathrm{Cl}$ & $\mathrm{I}_{2}-\mathrm{O}-$ & $-\mathrm{H}$ & $-\mathrm{CH}_{3}$ & $-\mathrm{H}$ & $-\mathrm{H}$ & $-\mathrm{OH}$ & $-\mathrm{OCH}_{3}$ & Hernandia sonora ${ }^{[164]}$ \\
\hline Launobine & $-\mathrm{O}-\mathrm{Cl}$ & $\mathrm{I}_{2}-\mathrm{O}-$ & $-\mathrm{H}$ & $-\mathrm{H}$ & $-\mathrm{H}$ & $-\mathrm{H}$ & $-\mathrm{OCH}_{3}$ & $-\mathrm{OH}$ & Illigera luzonensis ${ }^{[54]}$ \\
\hline Bulbocapnine & $-\mathrm{O}-\mathrm{Cl}$ & $\mathrm{I}_{2}-\mathrm{O}-$ & $-\mathrm{H}$ & $-\mathrm{CH}_{3}$ & $-\mathrm{H}$ & $-\mathrm{H}$ & $-\mathrm{OCH}_{3}$ & $-\mathrm{OH}$ & $\begin{array}{l}\text { Corydalis cava Schweigg. \& } \text { Kort }^{[151]} \\
\text { Cassytha filiformis }{ }^{[119]} \\
\text { Hernandia nymphaeifolia }{ }^{[128]} \\
\text { Illigera luzonensis }^{[135]} \\
\text { Corydalis hsuchowensis }^{[165]} \\
\text { Fumaria parviflora }^{[166]} \\
\text { Corydalis slivenensis }^{[104]} \\
\text { Corydalis bulbosa }^{[106]} \\
\text { Corydalis marschalliana }^{[111]}\end{array}$ \\
\hline O-Methylbulbocapnine & \multicolumn{2}{|c|}{$-\mathrm{O}-\mathrm{CH}_{2}-\mathrm{O}-$} & $-\mathrm{H}$ & $-\mathrm{CH}_{3}$ & $-\mathrm{H}$ & $-\mathrm{H}$ & $-\mathrm{OCH}_{3}$ & $-\mathrm{OCH}_{3}$ & $\begin{array}{l}\text { Hernandia nymphaeifolia }{ }^{[167]} \\
\text { Illigera luzonensis }^{[54]}\end{array}$ \\
\hline Ovigerine & \multicolumn{2}{|c|}{$-\mathrm{O}-\mathrm{CH}_{2}-\mathrm{O}-$} & $-\mathrm{H}$ & $-\mathrm{H}$ & $-\mathrm{H}$ & $-\mathrm{H}$ & \multicolumn{2}{|c|}{$-\mathrm{O}-\mathrm{CH}_{2}-\mathrm{O}-$} & $\begin{array}{l}\text { Hernandia nymphaeifolia }{ }^{[168]} \\
\text { Hernandia sonora }^{[164]}\end{array}$ \\
\hline N-Methylovigerine & \multicolumn{2}{|c|}{$-\mathrm{O}-\mathrm{CH}_{2}-\mathrm{O}-$} & $-\mathrm{H}$ & $-\mathrm{CH}_{3}$ & $-\mathrm{H}$ & $-\mathrm{H}$ & \multicolumn{2}{|c|}{$-\mathrm{O}-\mathrm{CH}_{2}-\mathrm{O}-$} & Hernandia nymphaeifolia ${ }^{[168]}$ \\
\hline Preocoteine ${ }^{[2]}$ & $-\mathrm{OH}$ & $-\mathrm{OCH}_{3}$ & $-\mathrm{H}$ & $-\mathrm{CH}_{3}$ & $-\mathrm{H}$ & $-\mathrm{OCH}_{3}$ & $-\mathrm{OCH}_{3}$ & $-\mathrm{H}$ & Ranuncudaceae: Thalictrum \\
\hline $\begin{array}{l}\text { Preocoteine } \mathrm{N} \text {-oxid }{ }^{[2]} \\
(\mathrm{N} \rightarrow \mathrm{O})\end{array}$ & $-\mathrm{OH}$ & $-\mathrm{OCH}_{3}$ & $-\mathrm{H}$ & $-\mathrm{CH}_{3}$ & $-\mathrm{H}$ & $-\mathrm{OCH}_{3}$ & $-\mathrm{OCH}_{3}$ & $-\mathrm{H}$ & Ranuncudaceae: Thalictrum \\
\hline O-Demethylpuepareine ${ }^{[2]}$ & $-\mathrm{OCH}_{3}$ & $-\mathrm{OCH}_{3}$ & $-\mathrm{OH}$ & $-\mathrm{CH}_{3}$ & $-\mathrm{H}$ & $-\mathrm{OCH}_{3}$ & $-\mathrm{OCH}_{3}$ & $-\mathrm{H}$ & Annonaceae: Annona \\
\hline Norpureine $^{[2]}$ & $-\mathrm{OCH}_{3}$ & $-\mathrm{OCH}_{3}$ & $-\mathrm{OCH}_{3}$ & $-\mathrm{H}$ & $-\mathrm{H}$ & $-\mathrm{OCH}_{3}$ & $-\mathrm{OCH}_{3}$ & $-\mathrm{H}$ & Annonaceae: Annona \\
\hline Thalicsimidine & $-\mathrm{OCH}_{3}$ & $-\mathrm{OCH}_{3}$ & $-\mathrm{OCH}_{3}$ & $-\mathrm{CH}_{3}$ & $-\mathrm{H}$ & $-\mathrm{OCH}_{3}$ & $-\mathrm{OCH}_{3}$ & $-\mathrm{H}$ & $\begin{array}{l}\text { Annona purpurea } \\
\text { Thalictrum flavum } \mathrm{L}^{[169]}\end{array}$ \\
\hline Oconovine & $-\mathrm{OCH}_{3}$ & $-\mathrm{OCH}_{3}$ & $-\mathrm{OCH}_{3}$ & $-\mathrm{CH}_{3}$ & $-\mathrm{H}$ & $-\mathrm{H}$ & $-\mathrm{OCH}_{3}$ & $-\mathrm{OH}$ & Thalictrum fauriei $i^{[170]}$ \\
\hline Ocokryptine $^{[2]}$ & $-\mathrm{OCH}_{3}$ & $-\mathrm{O}-\mathrm{CH}$ & $\mathrm{I}_{2}-\mathrm{O}-$ & $-\mathrm{CH}_{3}$ & $-\mathrm{H}$ & $-\mathrm{H}$ & $-\mathrm{OCH}_{3}$ & $-\mathrm{OH}$ & Lauraceae: Coctea \\
\hline Cassythine & \multicolumn{2}{|c|}{$-\mathrm{O}-\mathrm{CH}_{2}-\mathrm{O}-$} & $-\mathrm{OCH}_{3}$ & $-\mathrm{H}$ & $-\mathrm{H}$ & $-\mathrm{OH}$ & $-\mathrm{OCH}_{3}$ & $-\mathrm{H}$ & $\begin{array}{l}\text { Cassytha filiformis }{ }^{[137]} \\
\text { Cassytha filiformis }{ }^{[171]} \\
\text { Cassytha filiformis }{ }^{[119]} \\
\text { Cassytha filiformis }{ }^{[134]}\end{array}$ \\
\hline O-Methylcassyfiline ${ }^{[2]}$ & \multicolumn{2}{|c|}{$-\mathrm{O}-\mathrm{CH}_{2}-\mathrm{O}-$} & $-\mathrm{OCH}_{3}$ & $-\mathrm{H}$ & $-\mathrm{H}$ & $-\mathrm{OCH}_{3}$ & $-\mathrm{OCH}_{3}$ & $-\mathrm{H}$ & $\begin{array}{l}\text { Lauraceae: Cassytha } \\
\text { Ranuncudaceae: Thalictrum }\end{array}$ \\
\hline Ocoteine & \multicolumn{2}{|c|}{$-\mathrm{O}-\mathrm{CH}_{2}-\mathrm{O}-$} & $-\mathrm{OCH}_{3}$ & $-\mathrm{CH}_{3}$ & $-\mathrm{H}$ & $-\mathrm{OCH}_{3}$ & $-\mathrm{OCH}_{3}$ & $-\mathrm{H}$ & $\begin{array}{l}\text { Cassytha filiformis } \text { II }^{[125]} \\
\text { Slavjanka Mountains }\end{array}$ \\
\hline Csaaythidine $\mathrm{e}^{[2]}$ & \multicolumn{2}{|c|}{$-\mathrm{O}-\mathrm{CH}_{2}-\mathrm{O}-$} & $-\mathrm{OCH}_{3}$ & $-\mathrm{H}$ & $-\mathrm{H}$ & \multicolumn{2}{|c|}{$-\mathrm{O}-\mathrm{CH}_{2}-\mathrm{O}-$} & $-\mathrm{H}$ & Lauraceae: Cassytha \\
\hline Ocopodine $^{[2]}$ & \multicolumn{2}{|c|}{$-\mathrm{O}-\mathrm{CH}_{2}-\mathrm{O}-$} & $-\mathrm{H}$ & $-\mathrm{CH}_{3}$ & $-\mathrm{OCH}_{3}$ & \multirow{2}{*}{\multicolumn{2}{|c|}{$-\mathrm{O}-\mathrm{CH}_{2}-\mathrm{O}-$}} & $-\mathrm{H}$ & Lauraceae: Coctea \\
\hline Bisnorthalphenine ${ }^{[2]}$ & $-\mathrm{CH}_{2-}^{-}$ & $-\mathrm{OCH}_{3}$ & $-\mathrm{H}$ & $-\mathrm{H}$ & $-\mathrm{H}$ & & & $-\mathrm{O}-$ & Ranuncudaceae: Thalictrum \\
\hline Thalphenine & $-\mathrm{CH}_{2-}^{-}$ & $-\mathrm{OCH}_{3}$ & $-\mathrm{H}$ & $\left(-\mathrm{CH}_{3}\right)_{2}$ & $-\mathrm{H}$ & \multicolumn{2}{|c|}{$-\mathrm{O}-\mathrm{CH}_{2}-\mathrm{O}-$} & $-\mathrm{O}-$ & $\begin{array}{l}\text { Thalictrum przewalskii }{ }^{[173]} \\
\text { Thalictrum revolutum }^{[174]}\end{array}$ \\
\hline N-Acetylanonaine & \multicolumn{2}{|c|}{$-\mathrm{O}-\mathrm{CH}_{2}-\mathrm{O}-$} & $-\mathrm{H}$ & $-\mathrm{COCH}_{3}$ & $-\mathrm{H}$ & $-\mathrm{H}$ & $-\mathrm{H}$ & $-\mathrm{H}$ & $\begin{array}{l}\text { Aromadendron elegans-Blume } \\
\text { Magnolia obovata }^{[175]}\end{array}$ \\
\hline Isopiline $^{[2]}$ & $-\mathrm{OH}$ & $-\mathrm{OCH}_{3}$ & $-\mathrm{OCH}_{3}$ & $-\mathrm{H}$ & $-\mathrm{H}$ & $-\mathrm{H}$ & $-\mathrm{H}$ & $-\mathrm{H}$ & Annonaceae: Isolona \\
\hline O-Methylisopiline ${ }^{[2]}$ & $-\mathrm{OCH}_{3}$ & $-\mathrm{OCH}_{3}$ & $-\mathrm{OCH}_{3}$ & $-\mathrm{H}$ & $-\mathrm{H}$ & $-\mathrm{H}$ & $-\mathrm{H}$ & $-\mathrm{H}$ & Magnoliaceae: Liriodendron \\
\hline 3-Methoxynuciferine ${ }^{[2]}$ & $-\mathrm{OCH}_{3}$ & $-\mathrm{OCH}_{3}$ & $-\mathrm{OCH}_{3}$ & $-\mathrm{CH}_{3}$ & $-\mathrm{H}$ & $-\mathrm{H}$ & $-\mathrm{H}$ & $-\mathrm{H}$ & Magnoliaceae: Liriodendron \\
\hline Tuliferoline $^{[2]}$ & $-\mathrm{OCH}_{3}$ & $-\mathrm{OCH}_{3}$ & $-\mathrm{OCH}_{3}$ & $-\mathrm{COCH}_{3}$ & $-\mathrm{H}$ & $-\mathrm{H}$ & $-\mathrm{H}$ & $-\mathrm{H}$ & Magnoliaceae: Liriodendron \\
\hline Norstephalagine $^{[2]}$ & \multicolumn{2}{|c|}{$-\mathrm{O}-\mathrm{CH}_{2}-\mathrm{O}-$} & $-\mathrm{OCH}_{3}$ & $-\mathrm{H}$ & $-\mathrm{H}$ & $-\mathrm{H}$ & $-\mathrm{H}$ & $-\mathrm{H}$ & Artabotrys maingayi \\
\hline Zenkerine & $-\mathrm{OH}$ & $-\mathrm{OCH}_{3}$ & $-\mathrm{H}$ & $-\mathrm{H}$ & $-\mathrm{H}$ & $-\mathrm{H}$ & $-\mathrm{OCH}_{3}$ & $-\mathrm{H}$ & Uvaria klaineana $^{[191]}$ \\
\hline Pulchine $^{[2]}$ & $-\mathrm{OH}$ & $-\mathrm{OCH}_{3}$ & $-\mathrm{H}$ & $-\mathrm{CH}_{3}$ & $-\mathrm{H}$ & $-\mathrm{H}$ & $-\mathrm{OCH}_{3}$ & $-\mathrm{H}$ & Lauraceae: Ocotea \\
\hline
\end{tabular}




\begin{tabular}{|c|c|c|c|c|c|c|c|c|c|}
\hline Zanthoxyphylline $^{[2]}$ & $-\mathrm{OCH}_{3}$ & $-\mathrm{OCH}_{3}$ & $-\mathrm{H}$ & $\left(-\mathrm{CH}_{3}\right)_{2}$ & $-\mathrm{H}$ & $-\mathrm{H}$ & $-\mathrm{H}$ & $-\mathrm{OCH}_{3}$ & Rutaceae: Zanthoxylum \\
\hline Norlaureline $^{[2]}$ & \multicolumn{2}{|c|}{$-\mathrm{O}-\mathrm{CH}_{2}-\mathrm{O}-$} & $-\mathrm{H}$ & $-\mathrm{H}$ & $-\mathrm{H}$ & $-\mathrm{H}$ & $-\mathrm{H}$ & $-\mathrm{OCH}_{3}$ & Guatteria elata \\
\hline Puterine $^{[2]}$ & \multicolumn{2}{|c|}{$-\mathrm{O}-\mathrm{CH}_{2}-\mathrm{O}-$} & $-\mathrm{H}$ & $-\mathrm{CH}_{3}$ & $-\mathrm{H}$ & $-\mathrm{H}$ & $-\mathrm{H}$ & $-\mathrm{OCH}_{3}$ & Guatteria elata \\
\hline Buxifoline $e^{[2]}$ & \multicolumn{2}{|c|}{$-\mathrm{O}-\mathrm{CH}_{2}-\mathrm{O}-$} & $-\mathrm{OCH}_{3}$ & $-\mathrm{H}$ & $-\mathrm{H}$ & $-\mathrm{OCH}_{3}$ & $-\mathrm{H}$ & $-\mathrm{H}$ & Annonaceae: Xylopia \\
\hline Elmerrillicine $^{[2]}$ & \multicolumn{2}{|c|}{$-\mathrm{O}-\mathrm{CH}_{2}-\mathrm{O}-$} & $-\mathrm{OCH}_{3}$ & $-\mathrm{H}$ & $-\mathrm{H}$ & $-\mathrm{H}$ & $-\mathrm{H}$ & $-\mathrm{OH}$ & Magnoliaceae: Elmerrillia \\
\hline Liriotulipiferine $^{[2]}$ & $-\mathrm{OCH}_{3}$ & $-\mathrm{OH}$ & $-\mathrm{H}$ & $-\mathrm{H}$ & $-\mathrm{H}$ & $-\mathrm{OCH}_{3}$ & $-\mathrm{OH}$ & $-\mathrm{H}$ & Magnoliaceae: Liriodendron \\
\hline Norisodomesticine & $-\mathrm{OCH}_{3}$ & $-\mathrm{OH}$ & $-\mathrm{H}$ & $-\mathrm{H}$ & $-\mathrm{H}$ & \multicolumn{2}{|c|}{$-\mathrm{O}-\mathrm{CH}_{2}-\mathrm{O}-$} & $-\mathrm{H}$ & Guatteria dumetorum ${ }^{[98]}$ \\
\hline Lirioferine & $-\mathrm{OCH}_{3}$ & $-\mathrm{OCH}_{3}$ & $-\mathrm{H}$ & $-\mathrm{CH}_{3}$ & $-\mathrm{H}$ & $-\mathrm{OCH}_{3}$ & $-\mathrm{OH}$ & $-\mathrm{H}$ & Guatteria dumetorum $^{[98]}$ \\
\hline N-Methylnantenine & $-\mathrm{OCH}_{3}$ & $-\mathrm{OCH}_{3}$ & $-\mathrm{H}$ & $\left(-\mathrm{CH}_{3}\right)_{2}$ & $-\mathrm{H}$ & \multicolumn{2}{|c|}{$-\mathrm{O}-\mathrm{CH}_{2}-\mathrm{O}-$} & $-\mathrm{H}$ & Thalictrum przewalskii ${ }^{[177]}$ \\
\hline Litseferine $^{[2]}$ & \multicolumn{2}{|c|}{$-\mathrm{O}-\mathrm{CH}_{2}-\mathrm{O}-$} & $-\mathrm{H}$ & $-\mathrm{H}$ & $-\mathrm{H}$ & $-\mathrm{OCH}_{3}$ & $-\mathrm{OH}$ & $-\mathrm{H}$ & Lauraceae: Litsea \\
\hline Nordicentrine & \multicolumn{2}{|c|}{$-\mathrm{O}-\mathrm{CH}_{2}-\mathrm{O}-$} & $-\mathrm{H}$ & $-\mathrm{H}$ & $-\mathrm{H}$ & $-\mathrm{OCH}_{3}$ & $-\mathrm{OCH}_{3}$ & $-\mathrm{H}$ & Stephania pierrei ${ }^{[70]}$ \\
\hline Litsedine $^{[2]}$ & \multicolumn{2}{|c|}{$-\mathrm{O}-\mathrm{CH}_{2}-\mathrm{O}-$} & $-\mathrm{H}$ & $-\mathrm{H}$ & $-\mathrm{H}$ & $-\mathrm{H}$ & $-\mathrm{OCH}_{3}$ & $-\mathrm{OCH}_{3}$ & Lauraceae: Litsea \\
\hline Delporphine $^{[2]}$ & $-\mathrm{OCH}_{3}$ & $-\mathrm{OCH}_{3}$ & $-\mathrm{OH}$ & $-\mathrm{CH}_{3}$ & $-\mathrm{H}$ & $-\mathrm{OH}$ & $-\mathrm{OCH}_{3}$ & $-\mathrm{H}$ & Ranunculaceae: Delphinium \\
\hline Noroconovine ${ }^{[2]}$ & $-\mathrm{OCH}_{3}$ & $-\mathrm{OCH}_{3}$ & $-\mathrm{OCH}_{3}$ & $-\mathrm{H}$ & $-\mathrm{H}$ & $-\mathrm{H}$ & $-\mathrm{OCH}_{3}$ & $-\mathrm{OH}$ & Annonaceae: Polyalthia \\
\hline Polygospermine ${ }^{[2]}$ & $-\mathrm{OCH}_{3}$ & $-\mathrm{OCH}_{3}$ & $-\mathrm{OCH}_{3}$ & $-\mathrm{H}$ & $-\mathrm{H}$ & $-\mathrm{H}$ & $-\mathrm{O}-\mathrm{CH}$ & $\mathrm{H}_{2}-\mathrm{O}-$ & Annonaceae: Polyalthia \\
\hline Leucoxine $^{[2]}$ & \multicolumn{2}{|c|}{$-\mathrm{O}-\mathrm{CH}_{2}-\mathrm{O}-$} & $-\mathrm{H}$ & $-\mathrm{CH}_{3}$ & $-\mathrm{OH}$ & $-\mathrm{OCH}_{3}$ & $-\mathrm{OCH}_{3}$ & $-\mathrm{H}$ & $\begin{array}{l}\text { Ocotea minarum } \\
\text { Ocotea brachybotra }\end{array}$ \\
\hline Ocoxylonine $e^{[2]}$ & \multicolumn{2}{|c|}{$-\mathrm{O}-\mathrm{CH}_{2}-\mathrm{O}-$} & $-\mathrm{OCH}_{3}$ & $-\mathrm{CH}_{3}$ & $-\mathrm{OH}$ & $-\mathrm{OCH}_{3}$ & $-\mathrm{OCH}_{3}$ & $-\mathrm{H}$ & Lauraceae: Ocotea \\
\hline Leucoxylonine $^{[2]}$ & \multicolumn{2}{|c|}{$-\mathrm{O}-\mathrm{CH}_{2}-\mathrm{O}-$} & $-\mathrm{OCH}_{3}$ & $-\mathrm{CH}_{3}$ & $-\mathrm{OCH}_{3}$ & $-\mathrm{OCH}_{3}$ & $-\mathrm{OCH}_{3}$ & $-\mathrm{H}$ & Ocotea minarum \\
\hline N-Demethylthalphenine ${ }^{[2]}$ & $-\mathrm{CH}_{2-}^{-}$ & $-\mathrm{OCH}_{3}$ & $-\mathrm{H}$ & $-\mathrm{CH}_{3}$ & $-\mathrm{H}$ & \multicolumn{2}{|c|}{$-\mathrm{O}-\mathrm{CH}_{2}-\mathrm{O}-$} & $-\mathrm{O}-$ & Ranunculaceae: Thalictrum \\
\hline $\begin{array}{l}\mathrm{N}-\text { Carbamoyl- } \\
\text { asimilobine }^{[3]}\end{array}$ & $-\mathrm{OCH}_{3}$ & $-\mathrm{OH}$ & $-\mathrm{H}$ & $-\mathrm{CONH}_{2}$ & $-\mathrm{H}$ & $-\mathrm{H}$ & $-\mathrm{H}$ & $-\mathrm{H}$ & Annonaceae: Hexalobus \\
\hline $\begin{array}{l}\text { Roemerine } \mathrm{N} \text {-oxide }{ }^{[3]} \\
(\mathrm{N} \rightarrow \mathrm{O})\end{array}$ & \multicolumn{2}{|c|}{$-\mathrm{O}-\mathrm{CH}_{2}-\mathrm{O}-$} & $-\mathrm{H}$ & $-\mathrm{CH}_{3}$ & $-\mathrm{H}$ & $-\mathrm{H}$ & $-\mathrm{H}$ & $-\mathrm{H}$ & Papaveraceae: Papaver \\
\hline N-Formylanonaine & \multicolumn{2}{|c|}{$-\mathrm{O}-\mathrm{CH}_{2}-\mathrm{O}-$} & $-\mathrm{H}$ & $-\mathrm{CHO}$ & $-\mathrm{H}$ & $-\mathrm{H}$ & $-\mathrm{H}$ & $-\mathrm{H}$ & Magnolia obovata ${ }^{[175]}$ \\
\hline N-Carbamoylanonaine ${ }^{[3]}$ & $-\mathrm{O}-\mathrm{Cl}$ & $\mathrm{H}_{2}-\mathrm{O}-$ & $-\mathrm{H}$ & $-\mathrm{CONH}_{2}$ & $-\mathrm{H}$ & $-\mathrm{H}$ & $-\mathrm{H}$ & $-\mathrm{H}$ & Annonaceae: Hexalobus \\
\hline Norlirdinine $^{[3]}$ & $-\mathrm{OCH}_{3}$ & $-\mathrm{OH}$ & $-\mathrm{OCH}_{3}$ & $-\mathrm{H}$ & $-\mathrm{H}$ & $-\mathrm{H}$ & $-\mathrm{H}$ & $-\mathrm{H}$ & Annonaceae: Polyaltbia \\
\hline 3-Hydroxynornuciferine & $-\mathrm{OCH}_{3}$ & $-\mathrm{OCH}_{3}$ & $-\mathrm{OH}$ & $-\mathrm{H}$ & $-\mathrm{H}$ & $-\mathrm{H}$ & $-\mathrm{H}$ & $-\mathrm{H}$ & Artabotrys hainanensis ${ }^{[12]}$ \\
\hline $\begin{array}{l}\text { 9-Hydroxy-1,2- } \\
\text { dimethoxynoraporphine }\end{array}$ & $-\mathrm{OCH}_{3}$ & $-\mathrm{OCH}_{3}$ & $-\mathrm{H}$ & $-\mathrm{H}$ & $-\mathrm{H}$ & $-\mathrm{OH}$ & $-\mathrm{H}$ & $-\mathrm{H}$ & Annonaceae: Monantbotaxis \\
\hline $\mathrm{N}$-formylxylopine $\mathrm{e}^{[3]}$ & $-\mathrm{O}-\mathrm{Cl}$ & $\mathrm{H}_{2}-\mathrm{O}-$ & $-\mathrm{H}$ & $-\mathrm{CHO}$ & $-\mathrm{H}$ & $-\mathrm{OCH}_{3}$ & $-\mathrm{H}$ & $-\mathrm{H}$ & Annonaceae: Duguetia \\
\hline $\begin{array}{l}\text { 1,2,10-Trimethoxy- } \\
\text { aporphine }^{[3]}\end{array}$ & $-\mathrm{OCH}_{3}$ & $-\mathrm{OCH}_{3}$ & $-\mathrm{H}$ & $-\mathrm{CH}_{3}$ & $-\mathrm{H}$ & $-\mathrm{H}$ & $-\mathrm{OCH}_{3}$ & $-\mathrm{H}$ & Ranunculaceae: Tbalictrum \\
\hline Isothebaidine $^{[3]}$ & $-\mathrm{OH}$ & $-\mathrm{OCH}_{3}$ & $-\mathrm{H}$ & $-\mathrm{CH}_{3}$ & $-\mathrm{H}$ & $-\mathrm{H}$ & $-\mathrm{H}$ & $-\mathrm{OH}$ & Papaveraceae: Papaver \\
\hline N-Formylputerine ${ }^{[3]}$ & $-\mathrm{O}-\mathrm{Cl}$ & $\mathrm{H}_{2}-\mathrm{O}-$ & $-\mathrm{H}$ & $-\mathrm{CHO}$ & $-\mathrm{H}$ & $-\mathrm{H}$ & $-\mathrm{H}$ & $-\mathrm{OCH}_{3}$ & Annonaceae: Duguetia \\
\hline N-Methylbuxifoline ${ }^{[3]}$ & $-\mathrm{O}-\mathrm{Cl}$ & $\mathrm{H}_{2}-\mathrm{O}-$ & $-\mathrm{OCH}_{3}$ & $-\mathrm{CH}_{3}$ & $-\mathrm{H}$ & $-\mathrm{OCH}_{3}$ & $-\mathrm{H}$ & $-\mathrm{H}$ & Annonaceae: Duguetia \\
\hline N-Formylbuxifoline ${ }^{[3]}$ & $-\mathrm{O}-\mathrm{Cl}$ & $\mathrm{H}_{2}-\mathrm{O}-$ & $-\mathrm{OCH}_{3}$ & $-\mathrm{CHO}$ & $-\mathrm{H}$ & $-\mathrm{OCH}_{3}$ & $-\mathrm{H}$ & $-\mathrm{H}$ & Annonaceae: Duguetia \\
\hline (-)Norannuradhapurine $\mathrm{e}^{[3]}$ & $-\mathrm{O}-\mathrm{Cl}$ & $\mathrm{H}_{2}-\mathrm{O}-$ & $-\mathrm{H}$ & $-\mathrm{H}$ & $-\mathrm{OH}$ & $-\mathrm{OCH}_{3}$ & $-\mathrm{H}$ & $-\mathrm{H}$ & Annonaceae: Polyaltbia \\
\hline Stesakine & $-\mathrm{O}-\mathrm{Cl}$ & $\mathrm{H}_{2}-\mathrm{O}-$ & $-\mathrm{H}$ & $-\mathrm{CH}_{3}$ & $-\mathrm{OCH}_{3}$ & $-\mathrm{OH}$ & $-\mathrm{H}$ & $-\mathrm{H}$ & Stephania cepharantha ${ }^{[179]}$ \\
\hline N-Methylboldine ${ }^{[3]}$ & $-\mathrm{OCH}_{3}$ & $-\mathrm{OH}$ & $-\mathrm{H}$ & $\left(-\mathrm{CH}_{3}\right)_{2}$ & $-\mathrm{H}$ & $-\mathrm{OH}$ & $-\mathrm{OCH}_{3}$ & $-\mathrm{H}$ & Menispermaceae: Cocculus \\
\hline Laetanine $^{[3]}$ & $-\mathrm{OCH}_{3}$ & $-\mathrm{OH}$ & $-\mathrm{H}$ & $-\mathrm{H}$ & $-\mathrm{H}$ & $-\mathrm{OH}$ & $-\mathrm{OCH}_{3}$ & $-\mathrm{H}$ & Lauraceae: Litsea \\
\hline Isocalycinine $^{[3]}$ & $-\mathrm{O}-\mathrm{Cl}$ & $\mathrm{H}_{2}-\mathrm{O}-$ & $-\mathrm{H}$ & $-\mathrm{H}$ & $-\mathrm{H}$ & $-\mathrm{OH}$ & $-\mathrm{H}$ & $-\mathrm{OCH}_{3}$ & Annonaceae: Guatteria \\
\hline Calycinine & $-\mathrm{O}-\mathrm{Cl}$ & $\mathrm{H}_{2}-\mathrm{O}-$ & $-\mathrm{H}$ & $-\mathrm{H}$ & $-\mathrm{H}$ & $-\mathrm{OCH}_{3}$ & $-\mathrm{H}$ & $-\mathrm{OH}$ & Daphniphyllum unnanense $e^{[179]}$ \\
\hline N-Methylcalycinine ${ }^{[3]}$ & $-\mathrm{O}-\mathrm{Cl}$ & $\mathrm{H}_{2}-\mathrm{O}-$ & $-\mathrm{H}$ & $-\mathrm{CH}_{3}$ & $-\mathrm{H}$ & $-\mathrm{OCH}_{3}$ & $-\mathrm{H}$ & $-\mathrm{OH}$ & Annonaceae: Duguetia \\
\hline Discoguattine $^{[3]}$ & $-\mathrm{O}-\mathrm{Cl}$ & $\mathrm{H}_{2}-\mathrm{O}-$ & $-\mathrm{H}$ & $-\mathrm{H}$ & $-\mathrm{H}$ & $-\mathrm{OCH}_{3}$ & $-\mathrm{H}$ & $-\mathrm{OCH}_{3}$ & Annonaceae: Guatteria \\
\hline $\begin{array}{l}\text { Corydine } \mathrm{N} \text {-oxide }{ }^{[3]} \\
(\mathrm{N} \rightarrow \mathrm{O})\end{array}$ & $-\mathrm{OH}$ & $-\mathrm{OCH}_{3}$ & $-\mathrm{H}$ & $-\mathrm{CH}_{3}$ & $-\mathrm{H}$ & $-\mathrm{H}$ & $-\mathrm{OCH}_{3}$ & $-\mathrm{OCH}_{3}$ & Papaveraceae: Glaucium \\
\hline Glaufine $^{[3]}$ & $-\mathrm{OCH}_{3}$ & $-\mathrm{OH}$ & $-\mathrm{H}$ & $-\mathrm{CH}_{3}$ & $-\mathrm{H}$ & $-\mathrm{H}$ & $-\mathrm{OH}$ & $-\mathrm{OH}$ & Papaveraceae: Glaucium \\
\hline Laetine & $-\mathrm{OCH}_{3}$ & $-\mathrm{OH}$ & $-\mathrm{H}$ & $-\mathrm{H}$ & $-\mathrm{H}$ & $-\mathrm{H}$ & $-\mathrm{O}-\mathrm{CH}$ & $\mathrm{H}_{2}-\mathrm{O}-$ & Hernandia nymphaeifolia ${ }^{[167]}$ \\
\hline Hernagine $^{[3]}$ & $-\mathrm{OCH}_{3}$ & $-\mathrm{OCH}_{3}$ & $-\mathrm{H}$ & $-\mathrm{H}$ & $-\mathrm{H}$ & $-\mathrm{H}$ & $-\mathrm{OH}$ & $-\mathrm{OCH}_{3}$ & Hernandiaceae: Hernandia \\
\hline $\begin{array}{l}\text { Isocorydine } \mathrm{N} \text {-oxide }^{[3]} \\
(\mathrm{N} \rightarrow \mathrm{O})\end{array}$ & $-\mathrm{OCH}_{3}$ & $-\mathrm{OCH}_{3}$ & $-\mathrm{H}$ & $-\mathrm{CH}_{3}$ & $-\mathrm{H}$ & $-\mathrm{H}$ & $-\mathrm{OCH}_{3}$ & $-\mathrm{OH}$ & Berberidaceae: Berberis \\
\hline $\begin{array}{l}\mathrm{N}, \mathrm{O}-\text { Dimethyl- } \\
\text { isocorydinw } \mathrm{w}^{[3]}\end{array}$ & $-\mathrm{OCH}_{3}$ & $-\mathrm{OCH}_{3}$ & $-\mathrm{H}$ & $\left(-\mathrm{CH}_{3}\right)_{2}$ & $-\mathrm{H}$ & $-\mathrm{H}$ & $-\mathrm{OCH}_{3}$ & $-\mathrm{OCH}_{3}$ & Menispermaceae: Cocculus,Pacbygone \\
\hline N-Methylbulbocapnine ${ }^{[3]}$ & $-\mathrm{O}-\mathrm{Cl}$ & $\mathrm{H}_{2}-\mathrm{O}-$ & $-\mathrm{H}$ & $\left(-\mathrm{CH}_{3}\right)_{2}$ & $-\mathrm{H}$ & $-\mathrm{H}$ & $-\mathrm{OCH}_{3}$ & $-\mathrm{OH}$ & Fumariaceae: Corydalis \\
\hline Isooconovine $^{[3]}$ & $-\mathrm{OH}$ & $-\mathrm{OCH}_{3}$ & $-\mathrm{OCH}_{3}$ & $-\mathrm{CH}_{3}$ & $-\mathrm{H}$ & $-\mathrm{H}$ & $-\mathrm{OCH}_{3}$ & $-\mathrm{OCH}_{3}$ & Lauraceae: Ocotea \\
\hline Xyloguyelline $e^{[3]}$ & $-\mathrm{OCH}_{3}$ & $-\mathrm{OH}$ & $-\mathrm{OCH}_{3}$ & $-\mathrm{H}$ & $-\mathrm{H}$ & $-\mathrm{O}-\mathrm{Cl}$ & $\mathrm{I}_{2}-\mathrm{O}-$ & $-\mathrm{H}$ & Annonaceae: Xylopia \\
\hline Danguyelline $^{[3]}$ & $-\mathrm{OCH}_{3}$ & $-\mathrm{OH}$ & $-\mathrm{OCH}_{3}$ & $-\mathrm{H}$ & $-\mathrm{H}$ & $-\mathrm{H}$ & $-\mathrm{OCH}_{3}$ & $-\mathrm{OH}$ & Annonaceae: Xylopia \\
\hline Thalisopynine $^{[3]}$ & $-\mathrm{OCH}_{3}$ & $-\mathrm{OCH}_{3}$ & $-\mathrm{OCH}_{3}$ & $-\mathrm{CH}_{3}$ & $-\mathrm{H}$ & $-\mathrm{OH}$ & $-\mathrm{OCH}_{3}$ & $-\mathrm{H}$ & Ranunculaceae: Tbalictrum \\
\hline Baicaline $^{[3]}$ & $-\mathrm{OCH}_{3}$ & $-\mathrm{O}-\mathrm{CH}$ & $\mathrm{I}_{2}-\mathrm{O}-$ & $-\mathrm{H}$ & $-\mathrm{H}$ & $-\mathrm{OCH}_{3}$ & $-\mathrm{OCH}_{3}$ & $-\mathrm{H}$ & Ranunculaceae: Tbalictrum \\
\hline Duguevanine $^{[3]}$ & $-\mathrm{O}-\mathrm{Cl}$ & $\mathrm{I}_{2}-\mathrm{O}-$ & $-\mathrm{OCH}_{3}$ & $-\mathrm{H}$ & $-\mathrm{H}$ & $-\mathrm{OCH}_{3}$ & $-\mathrm{H}$ & $-\mathrm{OH}$ & Annonaceae: Duguetia \\
\hline N-Methylduguevanine ${ }^{[3]}$ & $-\mathrm{O}-\mathrm{Cl}$ & $\mathrm{H}_{2}-\mathrm{O}-$ & $-\mathrm{OCH}_{3}$ & $-\mathrm{CH}_{3}$ & $-\mathrm{H}$ & $-\mathrm{OCH}_{3}$ & $-\mathrm{H}$ & $-\mathrm{OH}$ & Annonaceae: Duguetia \\
\hline N-Formylduguevanine ${ }^{[3]}$ & $-\mathrm{O}-\mathrm{Cl}$ & $\mathrm{H}_{2}-\mathrm{O}-$ & $-\mathrm{OCH}_{3}$ & $-\mathrm{CHO}$ & $-\mathrm{H}$ & $-\mathrm{OCH}_{3}$ & $-\mathrm{H}$ & $-\mathrm{OH}$ & Annonaceae: Duguetia \\
\hline Ocominarine $^{[3]}$ & $-\mathrm{O}-\mathrm{Cl}$ & $\mathrm{H}_{2}-\mathrm{O}-$ & $-\mathrm{H}$ & $-\mathrm{CH}_{3}$ & $-\mathrm{O}-\mathrm{C}$ & $\mathrm{I}_{2}-\mathrm{O}-$ & $-\mathrm{OCH}_{3}$ & $-\mathrm{H}$ & Ocotea minarum \\
\hline Norleucoxylonine $^{[3]}$ & $-\mathrm{O}-\mathrm{Cl}$ & $\mathrm{H}_{2}-\mathrm{O}-$ & $-\mathrm{OCH}_{3}$ & $-\mathrm{H}$ & $-\mathrm{OCH}_{3}$ & $-\mathrm{OCH}_{3}$ & $-\mathrm{OCH}_{3}$ & $-\mathrm{H}$ & Lauraceae: Ocotea \\
\hline Ocotominarine $^{[3]}$ & $-\mathrm{O}-\mathrm{Cl}$ & $\mathrm{H}_{2}-\mathrm{O}-$ & $-\mathrm{OCH}_{3}$ & $-\mathrm{CH}_{3}$ & $-\mathrm{O}-\mathrm{C}$ & $\mathrm{I}_{2}-\mathrm{O}-$ & $-\mathrm{OCH}_{3}$ & $-\mathrm{H}$ & Ocotea minarum \\
\hline
\end{tabular}


with $\alpha_{1}$ B-receptor. Concentration has great effects on $(+/-)$ domesticine's ${ }^{181}$ selection of receptor and pharmacological effects of (+)-nantenine observed at concentrations lower than $1 \mu \mathrm{M}$ can be attributed to $\alpha_{1}$-adrenergic and 5-HT 2A receptor blocking properties whereas at higher concentrations $(>1 \mu \mathrm{M})$ the pharmacological activity of this natural compound may be also due to a decrease of $\mathrm{Ca}^{2+}$ influx through transmembrane calcium channels (calcium antagonist activity), to an inhibition of protein kinase $\mathrm{C}(\mathrm{PKC})$ actions and/or to an $\alpha_{2}$-adrenoceptor blockade ${ }^{185}$. In addition, the substitution of aporphine alkaloids has important effects on pharmacological activity on blocking adrenoreceptor and selectivity for different subtypes. Hydroxy at the C-2 of boldine induces a significant increase in $\alpha_{1} \mathrm{~A}$ subtype selectivity and affinity ${ }^{186,187}$. The replacement of a methoxy moiety at $\mathrm{C}-1$ position of $( \pm)$-nantenine with a hydroxyl group increased affinity for the receptor ${ }^{188}$. A hydroxyl group at the $\mathrm{C}-1$ position and a methyl group at the $\mathrm{N}-6$ position in the $( \pm)$-nantenine structure are essential for the enhancement of affinity for the $\alpha_{1}$-adrenoceptor ${ }^{189}$.

Pharmacological activity on serotonin: Nantenine inhibits 1-5-HTP plus clorgyline-induced head- twitch response by blocking 5-HT2A receptors in the central nervous system ${ }^{189}$. (+)-Nantenine can inhibit 5-HT(2A) receptor to lower blood pressure and reduce heart rates ${ }^{191,192}$. Results suggest that a methyl group at a nitrogen atom and a methoxy moiety at $\mathrm{C}-1$ in (+)-nantenine play important roles in the development of the antiserotonergic activity ${ }^{190}$.

Cytotoxic activity: Cytotoxic activity is another important physiological activity of aporphine alkaloids. Alkaloids with cytotoxic activity have been shown in references ${ }^{15-22}$. Mechanism of this cytotoxic activity is that alkaloids with methylenedioxy ring groups interfere with the catalytic activity of topoisomerases in contrast other aporphines. These interactions with DNA may explain, at least in part, the effects observed on cancer cells and on trypanosomes ${ }^{119}$. The DNA damaging activity of Stephania dinklagei ${ }^{194}$ and Topoisomerase II inhibition by (+) dicentrine ${ }^{195}$ are the mechanism of this cytotoxic activity. (-)-Roemerine, isolated from the leaves of Annona senegalensis, was found to enhance the cytotoxic response mediated by vinblastine with multidrug-resistant KB-V1 cells. In the absence of vinblastine, no significant cytotoxicity was observed with $\mathrm{KB}-3$ or $\mathrm{KB}-\mathrm{V} 1$ cells $\left(\mathrm{ED}_{50}>\right.$ $20 \mu \mathrm{g} / \mathrm{mL}$ ) and several other human tumor cell lines were also relatively insensitive. As indicated by its ability to inhibit ATPdependent $[3 \mathrm{H}]$ vinblastine binding to multidrug-resistant KBV1 cell membrane vesicles, (-)-roemerine appears to function by interacting with P-glycoprotein ${ }^{89}$. In addition, different alkaloids have various sensibilities of different cells and substituent groups on alkaloids have effects on the cytotoxic activity.

Pharmacological activity on antioxidation: Boldine has been most widely studied in the pharmacological activity on antioxidation. Boldine inhibits the oxidation of LDL in vitro and atherosclerosis in vivo ${ }^{196}$, prevents human liver microsomal lipid peroxidation and inactivation of cytochrome P4502E $1^{197}$ and inhibits oxidative mitochondrial damage to prevent diabetes ${ }^{198}$. Boldine may attenuate the catecholamine oxidation-induced brain mitochondrial dysfunction and decrease the dopamine-induced death of PC12 cells through a scavenging action on reactive oxygen species and inhibition of melanin formation and thiol oxidation ${ }^{199}$. Many other aporphine alkaloids, such as isoboldine, Bulbocapnine, apomorphine, glaucine, magnoflorine and anonaine ${ }^{200,203}$, can take effect on antioxidation by removing active oxygen and active free radicles and inhibiting Hypoxanthine-xanthine oxidase system. Antioxidation of aporphine alkaloids is related to their structures ${ }^{203,204}$.

Pharmacological activity on antiplatelet effects: Many aporphine alkaloids have this pharmacological activity. The inhibitory effect of dicentrine on platelet aggregation and ATP release was due to the inhibition of thromboxane formation and the elevation of the level of cyclic $\mathrm{AMP}^{205}$. The antiplatelet effect of phenanthrene alkaloids is mainly a result of inhibition of thromboxane A2 formation ${ }^{206}$. In addition, sonodione, demethylsonodione, norsonodione, ovigerine, hernangerine, $\mathrm{N}$-methylhernangerine, (+)-malekulatine and isovanillin have obvious effects on antiplatelet effects ${ }^{125,207,208}$.

Pharmacological activity on nervous system: (S)-(+)Boldine was brominated, chlorinated and iodinated using molecular bromine in acetic acid or N-halosuccinimides in trifluoroacetic acid. Initial halogenation occurs at C-3, followed (in the cases of chlorine and bromine) by the less reactive $\mathrm{C}-8$, to afford 3-haloboldines- and 3,8-dihaloboldines. Using a 2:1 ratio of $\mathrm{N}$-iodosuccinimide to boldine, however, only the 3 -iodo derivative 6 was obtained. Radioligand binding studies of these products showed that halogenation of boldine at C-3 favours affinity for $\mathrm{D}(1)-[v s . \mathrm{D}(2)-]$ dopaminergic receptors, attaining a low nanomolar $\mathrm{IC}_{50}$ value in the case of 3 -iodoboldine ${ }^{209}$. Boldine does not display effective central dopaminergic antagonist activities in vivo in spite of its good binding affinity at D1- and D2-like receptors and that glaucine, although less effective in vitro, does appear to exhibit some antidopaminergic properties in vivo ${ }^{210}$. When tested on dopamine (DA) metabolism in the striatum of B6CBA mice, glaucine, 3-bromoboldine, glaucine, 3-bromoboldine, 3iodoboldine, 8-aminoboldine, 8-nitrosoboldine and 2,9-O, $\mathrm{O}^{\prime}$ dipivaloylboldine, increased striatal levels of DOPAC and HVA and the HVA/DA ratio, indicating that they cross the bloodbrain barrier and that they seem to act as dopamine antagonists in vivo ${ }^{211}$. The neuromuscular blockade by boldine on isolated mouse phrenic-nerve diaphragm might be due to its direct interaction with the postsynaptic nicotinic acetylcholine receptor $^{212}$. Corydine, isocorydine and glaucine's possible contributions to activity be preferential binding to blood components or by selective inhibition of acetylcholinesterase. The combined studies indicated that there was a modest preference by the neuromuscular junction of the cat for monoquaternary blockers with the (s)-confirguation ${ }^{213}$. Atherosperminine produced effects associated with dopamine receptor stimulation $^{214}$. Nantenine blocked and rapidly reversed MDMA-induced hyperthermia, attenuated lethality in both housing conditions and reduced MDMA-induced locomotor stimulation and head twitches ${ }^{215}$.

Immunoregulatory activity: Boldine concentrationdependently decreased blastogenesis in normal subjects and patients with chronic lymphocytic leukemia (CLL). However, 
the decrease in breast cancer patients was significant only at higher concentrations. Natural killer (NK) activity showed no change in healthy controls with normal values, but in cases with low activity treatment with boldine resulted in an increase. In patients with CLL, NK activity was enhanced; in tumorbearing patients, however, there was no effect. LDCC and NCMC activity did not change significantly in normal controls ${ }^{216}$. The inhibitory effect of 15 semi-synthetic analogues of glaucine on the lipopolysaccharide (LPS)-induced and the concanavalin A (Con A)-induced proliferation of mouse splenocytes was compared in vitro. Isoboldine, bracteoline and dehydroglaucine showed a significantly higher potency to suppress LPS-induced proliferation than 1, while 7-hydroxy-4-methylglaucine, 7formyldehydroglaucine, 7-acetyldehydroglaucine, 7-benzoyldehydroglaucine, oxoglaucine and glaucine-quinol were less inhibitory. Compounds 3, 4, boldine, 15 and 16 surpassed significantly the inhibition expressed by $\mathbf{1}$ on Con A-induced proliferative response $\mathrm{e}^{217}$. Oxoglaucine has strong effects on immunosuppression $^{218,219}$.

Pharmacological activity on antivirus: The pharmacological activity on antivirus of aporphine alkaloids is related to their structures. A series of 18 aporphinoids have been tested in vitro against human poliovirus. The aporphines (+)-glaucine fumarate, (+)-N-methyllaurotetanine, $(+)$-isoboldine and (-)nuciferine, $\mathrm{HCl}$ were found to be active with selectivity indices $>14$. The nature of the 1,2-substituents of the isoquinoline moiety appeared to be critical for antipoliovirus activity. An SAR study demonstrated the importance of a methoxyl group at C-2 on the tetrahydroisoquinoline ring for the induction of antipoliovirus activity. Molecular modeling of some compounds in this series revealed the close similarities between the threedimensional conformational features of the inactive 1,2-substituted derivatives (+)-boldine and (+)-laurolitsine with derivatives containing the 1,2-(methylenedioxy) moiety, which were generally found to be inactive as exemplified by (+)cassythicine ${ }^{220}$. Hernandonine, laurolistine, 7-oxohernangerine and lindechunine A showed significant anti-human immunodeficiency virus type 1 (HIV-1) integrase activity with IC values of $16.3,7.7,18.2$ and $21.1 \mu \mathrm{M}$, respectively ${ }^{221}$.

Pharmacological activity on ion channel: The pharmacological activity on ion channel of aporphine alkaloids mainly means that they can inhibit the calcium ion channels. (+)-Boldine $^{222}$, nantenine ${ }^{185,222,223}$, glaucine ${ }^{224}$ and laurotetanine ${ }^{43}$ relaxed the rat thoracic aorta mainly by suppressing the $\mathrm{Ca}^{2+}$ influx through both voltage- and receptor-operated calcium channels. Boldine could sensitize the ryanodine receptor and induce $\mathrm{Ca}^{2+}$ release from the internal $\mathrm{Ca}^{2+}$ storage site of skeletal muscle ${ }^{2}$. Boldine produces a non-parallel shift of the contraction response induced by barium, revealing the occurrence of a non-competitive antagonism. This effect could be the result of interference with intracellular events associated with the barium-induced changes in calcium pools ${ }^{227}$. In addition, some Aporphine alkaloids have effects on the ion channels of $\mathrm{K}^{+}, \mathrm{Na}^{+}, \mathrm{Ca}^{2+}$, for example, isocorydine may interfere with $\mathrm{K}^{+}, \mathrm{Na}^{+}$and $\mathrm{Ca}^{2+}$ currents in ventricular cells membrane at different concentrations ${ }^{228}$ and dicentrine has blocking effects on ion channels of $\mathrm{K}^{+}$and $\mathrm{Na}^{+}$currents in ventricular cells ${ }^{229,230}$.
Others: Aporphine alkaloids have other less common pharmacological activities. (S)-dicentrine and (S)-neolitsine have anthelmintic activity ${ }^{231}$. From the leaves of Nelumbonucifera (an aquatic plant), one new compound, 24(R)-ethylcholest-6ene-5 $\alpha$-ol-3- $O$ - $\beta$-D-glucopyranoside, along with 11 known metabolites, were isolated and identified by spectroscopic methods including 1D- and 2D NMR. Antifungalactivity for $(R)$-roemerine $\left(\mathrm{IC}_{50} / \mathrm{MIC}=4.5 / 10 \mu \mathrm{g} / \mathrm{mL}\right.$ against Candida albicans) and antimalarialactivity for $(R)$-roemerine and $N$-methylasimilobine $\left(\mathrm{IC}_{50}=0.2\right.$ and $4.8 \mu \mathrm{g} / \mathrm{mL}$ for the D6 clone, respectively and 0.4 and $4.8 \mu \mathrm{g} / \mathrm{mL}$ for the $\mathrm{W} 2$ clone, respectively) was observed. An analysis of the structureactivity relationship shows that the substituents in position C-1 and C-2 of aporphine alkaloids are crucial for the antimalarialactivity ${ }^{232}$. The alkaloids boldine, glaucine, predicentrine, apomorphine, coclaurine, norarmepavine and codeine may act by blocking mitochondrial electron transport. The trypanocidal effects of these alkaloids appear to be correlated with their antioxidative activities ${ }^{233}$. Boldine has antileishmanial, antiinflammatory, antipyretic, cytoprotective effects $^{234,235}$. (+)-Actinodaphnine, (+)-N-Me-actinodaphnine, (+)-anonaine, (-)-xylopine and (-)-N-Me-xylopine MeI, had the strongest inhibitory activities against three $\mathrm{G}(+)$ bacteria (Bacillus cereus, Micrococcus sp. and Staphylococcus aureus). (+)-Actinodaphnine, (+)-N-Me-actinodaphnine, (+)-anonaine, anhydroushinsunine, anhydroushinsunine MeI, ushinsunine isomethine, O-Me-armepavine methine and O,O-di-Et-N-Mecoclaurine methine had potent antifungal activities against Candida albicans, Cryptococcus neoformans and other Candida species $^{237,238}$.

\section{ACKNOWLEDGEMENTS}

The work was supported by the National Basic Research Program of China (973 Program) under Grants No. 2011CB505106, National Science Foundation of China under Grant No. 81173463 and 81102730. The New Century Excellent Talent support plan of the ministry of education under grant No. NCET-12-0804 and NCET-11-0607, The Beijing Nova Program under grant No. xx2013032 and 2011069, The Beijing Common special construction projects, The Foundation of Beijing University of Chinese Medicine of Education Ministry of China under grant No. 2011-CXTD-06.

\section{REFERENCES}

1. H.M. Guinaudeau, M. Leboeuf and A. Cave, Lloydia, 38, 275 (1975).

2. H.M. Guinaudeau, M. Leboeuf and A. Cave, J. Nat. Prod., 42, 325 (1979).

3. H.M. Guinaudeau, M. Leboeuf and A. Cave, J. Nat. Prod., 46, 761 (1983).

4. D. Cortes, R. Hocquemiller and M. Leboeuf, Phytochemistry, 24, 2776 (1985).

5. M. Sharaf and L.K. Wong, Phytochemistry, 29, 1899 (1990).

6. J.H. Wu and S.X. Liao, J. Modern Appl. Pharm., 11, 41 (1994).

7. N. Xie, R.N. Xu and S.M. Zhong, J. China Pharm. Univ., 25, 205 (1994).

8. L.J. Tian, N.Y. Yang and Z.G. Meng, J. Chin. Pharm. Sci., 38, 258 (2003).

9. Q.Z. Wang, M.F. He and J.Y. Liang, Chin. Herbal Med., 34, 277 (2003).

10. X.S. Peng, Y.H. Gao and Y.M. Diao, Jiangxi Inst. Tradit. Chin. Med. J., 16, 79 (2004) 
11. X.T. Liu, Q. Zhang and J.Y. Liang, Chin. J. Nat. Med., 2, 205 (2004).

12. C.G. Han and G.Y. Chen, J. Chin. Mater. Med., 30, 1660 (2005).

13. X.S. Peng, Y.H. Gao and Y.M. Diao, Chin. Herbal Med., 37, 984 (2006).

14. C.Y. Bo, W.Q. Yi and Z.L. Zhou, Chin. Med. Crop., 30, 409 (2007).

15. M. H, E. A and L. P, Phytochemistry, 68, 1813 (2007)

16. S. Kanokmedhakul, K. Kanokmedhakul and R. Lekphrom, J. Nat. Prod., 70, 1536 (2007).

17. Z.D. Min and S.M. Zhong, Acta Pharmacol. Sin., 15, 532 (1980).

18. C.Y. Hou and H. Xue, Acta Pharmacol. Sin., 19, 471 (1984).

19. C.Y. Hou and H. Xue, Acta Pharmacol. Sin., 20, 112 (1985).

20. H.S. Chen, H.Q. Liang and Z.L. Zhou, The Second Military Medical University Press, 7, 349 (1986).

21. C. Deng, T.F. Zhao and X.K. Wang, West China J. Pharm. Sci., 3, 65 (1988).

22. Y. Chen, S.D. Fang and J. Dai, J. Integrat. Plant Biol., 31, 544 (1989).

23. D.Y. Si and S.X. Zhao, J. Jining Med. College, 14, 1 (1991).

24. Y.Y. Chen, C.H. Qiu and L. Shen, J. Beijing Med. Univ., 23, 235 (1991).

25. J. Maria, B. Filho, E.V.L,D.Cunha, Phytochemistry, 44, 959 (1997).

26. Y. Deng, T.J. Tang and X. Li, Nat. Prod. Res., 21, 28 (2007).

27. Y.M. Hu and S.X. Zhao, Nanjing Coll. Pharm., 16, 7 (1985).

28. S.Y. Liu, West China J. Pharm. Sci., 2, 173 (1987).

29. S. Huang and F.K. Du, Hunan Guiding J. TCMP., 8, 582 (2002).

30. A. Langlois, D.A. Mulholland and N.R. Crouch, Biochem. Systemat. Ecol., 32, 1087 (2004).

31. W.S. Wang, W. Xiao and R. Yu, The Central University for Nationalities Journal (Natural Sciences), 16, 80 (2007).

32. J.F. Zheng and M.J. Qian, World Notes Plant Med., 22, 55 (2007).

33. H.F. Hao, L.J. Ren and Y.W. Chen, Acta Pharmacol. Sin., 31, 689 (1996).

34. Z.Y. Tian and Z.G. Li, Clockwise GuoYi Enterprise, 15, 704 (2004).

35. H.S. Chen, G.Y. Han and M.Z. Liu, The Second Military Medical University Press, 13, 167 (1992).

36. S.B. Chen, L.W. Wang and J.S. Yang, Chin. Herbal Med., 32, 3 (2001).

37. W.D. Zhang, G.Y. Han and H.Q. Liang, Sichuan Jiangyou Aconiti Alkaloids Ingredients, 27, 670 (1992).

38. G.Y. Gao, L.W. Wang and S.B. Chen, J. Chin. Pharm. Sci., 34, 157 (1999).

39. H. Wagner, L.-Z. Lin and O. Seligmann, Tetrahedron, 40, 2133 (1984).

40. M.P. Velcheva, S. Danghaaghiin and Z. Amdanghiin, Phytochemistry, 39, 683 (1995).

41. M.P.V. Rumiana and R. Petrova, Phytochemistry, 42, 535 (1996).

42. X.H. Yan, X.Y. Wei and H.H. Xie, Trop. Subtrop. Plants J., 8, 324 (2000).

43. S.Q. Chen and T. Zuo, Henan Univ. J. Tradit. Chin. Med., 20, 25 (2005).

44. L. Luo, J.K. Tian and S.L. Yang, Asia-Pacific Tradit. Med., 3, 57 (2007).

45. C.G.X,N. Nakamura, M.C.M, Chin. J. Nat. Med., 3, 275 (2005).

46. Z.Q. Zhao, Y.M. Zhao and K.J. Wang, Acta Pharmacol. Sin., 40, 931 (2005).

47. M.R. Mukhtar, M.T. Martin and M. Domansky, Phytochemistry, 45, 1543 (1997)

48. C.O. Castro, V. José López and G.A. Vergara, Phytochemistry, 24, 203 (1985).

49. J.H.Y. Vilegas, O.R. Gottlieb and M.A.C, Phytochemistry, 28, 3577 (1989)

50. X.K. Li and L.J. Wu, Chin. Herbal Med., 33, 676 (2002).

51. S.W. Yin, X.M. He and F.X. Lang, Guizhou Agric. Sci., 35, 133 (2007).

52. G.Y. Gu and L. Jiang, World Notes Plant Med., 20, 185 (2005).

53. F.A. Hussaini and A. Shoeb, Phytochemistry, 24, 633 (1985).

54. I.S. Chen and J.J. Chen, Planta Med., 63, 154 (1997).

55. Y. Wang,Y. Ju and S. Wang, Chin. Herbal Med., 33, 666 (2002).

56. J.H. Dou, J.S. Li and W.Z. Yan, J. Chin. Mater. Med., 14, 40 (1989).

57. G.Q. Xiao, X.Y. Lu and Y. Tian, Chem. Biol. Eng., 23, 1 (2006)

58. X. Zhang and Y. Fan, Nanjing Univ. Chin. Med. J., 18, 382 (2002).

59. G.Y. Liang, Y. Zhou and P.X. Cao, J. Chin. Pharm. Sci., 40, 900 (2005).

60. Z. Zheng, J. Chen and B. Chen, Nat. Prod. Res. Develop., 15, 322 (2003).

61. X.Y. Fu, W.Z. Liang and G.T. Xu, Acta Pharmacol. Sin., 21, 447 (1986).

62. A. Fournet, M.E. Ferreira and A. Rojas de Arias, Fitoterapia, 78, 382 (2007).

63. C. FR, W. JL, T.CM, Phytochemistry, 49, 2015 (1998).

64. S.N, U.A, S.N, J. Nat. Prod., 50, 773 (1987).

65. J.D. Phillipson, O.O. Thomas and A.I. Gray, Planta Med., 41, 105 (1981).
66. Y. Hong, X.L. Zhou and S.H. Huang, West China J. Pharm. Sci., 22, 30 (2007).

67. D. Zhen, H.C. Zheng and H.Q. Wang, Foreign Med. Plants Vol. Med., 12, 16 (1997)

68. E.J. Wang, Y.B. Ma and X.M. Zhang, Zhongguo Zhong Yao Za Zhi., 33, 2503 (2008).

69. J.A. Hasrat, T. De Bruyne and J.P. De Backer, J. Pharm. Pharmacol., 49, 1145 (1997).

70. K. Likhitwitayawuid, C.K. Angerhofer and H. Chai, J. Nat. Prod., 56, 1468 (1993).

71. Z. Xue, P.L. Zhang and J.M. Ma, Acta Pharmacol. Sin., 21, 223 (1986).

72. Z.P. Zheng, J.Y. Liang and L.H. Hu, Chin. J. Nat. Med., 3, 151 (2005).

73. G. Sariyar, Planta Med., 46, 175 (1982).

74. S. El-Masry, M.G. El-Ghazooly and A.A. Omar, Planta Med., 41, 61 (1981).

75. S.G. Zelenski, J. Pharm. Sci., 66, 1627 (1977).

76. H. Montenegro, M. Gutiérrez and L.I. Romero, Planta Med., 69, 667 (2003).

77. F.C. Ohiri, R. Verpoorte and A.B. Svendsen, Planta Med., 46, 228 (1982).

78. J.L. Wang, X.M. Hu and W.H. Yi, China J. Chin. Mater. Med., 16, 673 (1991).

79. Q. Paulo Mde, J.M. Barbosa-Filho and E.O. Lima, J. Ethnopharmacol., 36, 39 (1992)

80. X.J. Yang, L.Z. Xu and N.J. Sun, Acta Pharmacol. Sin., 27, 185 (1992).

81. D. Cortes, M.Y. Torrero and M. Pilar D'Ocon, J. Nat. Prod., 53, 503 (1990).

82. V. Mahiou, F. Roblot and R. Hocquemiller, J. Nat. Prod., 57, 890 (1994).

83. V. Muñoz, M. Sauvain and P. Mollinedo, Planta Med., 65, 448 (1999).

84. A.C. Gören, B.N. Zhou and D.G. Kingston, Planta Med., 69, 867 (2003).

85. J.T. Blanchfield, D.P. Sands and C.H. Kennard, Phytochemistry, 63, 711 (2003)

86. J.M. Huang and J.P. Luo, Chin. Med. Crop., 28, 186 (2005).

87. I.M. Fechine, J.F. Tavares and M.S. da Silva, Fitoterapia, 74, 29 (2003).

88. S. Chuliá, M.D. Ivorra and A. Cavé, J. Pharm. Pharmacol., 47, 647 (1995).

89. M. You, D.B. Wickramaratne and G.L. Silva, J. Nat. Prod., 58, 598 (1995).

90. Hussain, J. Nat. Prod., 49, 538 (1986).

91. X.K. Wang and T.F. Zhao, West China J. Pharm. Sci., 5, 39 (1990).

92. R. Sotníková, V. Kettmann and D. Kostálová, Methods Find Exp. Clin. Pharmacol., 19, 589 (1997).

93. G. Sanyar, H.B. Gülgeze and B. Gözler, Planta Med., 58, 368 (1992).

94. J.D. Phillipson, A. Scutt and A. Baytop, Pseudo-orientale, 43, 261 (1981).

95. Z.L. Zhang and S.Y. Ma, Yunnan Inst. Tradit. Chin. Med. J., 26, 52 (2003).

96. P.W. Le Quesne and J.E. Larrahondo, J. Nat. Prod., 43, 353 (1980).

97. T. Feng, Y. Xu, X.H. Cai and Z.Z. Du, Planta Med., 75, 76 (2009).

98. J.E. Correa, C.H. Ríos and A. del Rosario Castillo, Planta Med., 72 , 270 (2006).

99. Q.Z. Zhao, Y.M. Zhao and K.J. Wang, Acta Pharmacol. Sin., 40, 931 (2005).

100. F.W. Lin, P.L. Wu and T.S. Wu, Chem Pharm Bull. (Tokyo), 49, 1292 (2001).

101. G.Z. Wei, Z.L. Wen and S.T. Guo, Planta Med., 53, 418 (1987).

102. M.A. El-Kawi, D.J. Slatkin and P.L. Schiff Jr, J. Nat. Prod., 47, 459 (1984).

103. D.S. Bhakuni and S. Gupta, Planta Med., 48, 52 (1983).

104. H.G. Kiryakov, E. Iskrenova and E. Daskalova, Planta Med., 44, 168 (1982).

105. A.A. Leslie Gunatilaka and S. Sotheeswaran, Planta Med., 43, 309 (1981).

106. H.G. Kiryakov, E. Iskrenova and B. Kuzmanov, Planta Med., 41, 298 (1981).

107. P. Cheng, Y.B. Ma and S.Y. Yao, Bioorg. Med. Chem. Lett., 17, 5316 (2007).

108. G.Y. Gu and Y. Jiang, Foreign Med.-Vol. Herbal Med., 10, 153 (1995).

109. D.J. Milanowski, R.E. Winter and M.P. Elvin-Lewis, J. Nat. Prod., 65 , 814 (2002).

110. C.M. Teng, S.M. Yu and S.S. Lee, Eur. J. Pharmacol., 233, 7 (1993).

111. H.G. Kiryakov, E. Iskrenova and B. Kuzmanov, Planta Med., 43, 51 (1981). 
112. K. He, J.L. Gao and G.S. Zhao, Chin. Herbal Med., 38, 1909 (2007). 113. K. Iwasa, T. Takahashi and Y. Nishiyama, J. Nat. Prod., 71, 1376 (2008). 114. Y.C. Qian, Poppy, Grassroots Chin. Med. J., 15, 48 (2001).

115. X.H. Yan, F.X. Zhang and X.Y. Wei, Chin. Med. Crop., 23, 234 (2000). 116. M.R. Mukhtar, A.N. Aziz and N.F. Thomas, Molecules, 14, 1227 (2009). 117. Z. Han, Y. Zheng, N. Chen and L. Luan, J. Chromatogr. A,12,76 (2008),

118. L.Y. Chung, M.W. Lo and M.R. Mustafa, Phytother. Res., 23, 330 (2009)

119. S. Hoet, C. Stévigny and S. Block, Planta Med., 70, 407 (2004)

120. G. Schmeda-Hirschmann, J.A. Rodriguez and C. Theoduloz, Free Radic. Res., 37, 447 (2003).

121. G.X. Chuang, Z.T. Wang and L.S. Xu, Chin. Wild Plant Resour., 18, 5 (1999).

122. D. Qiu, L.X. Zhou and Y. Ding, Chin. Pharm. J., 36, 185 (2001).

123. N.T. Nguyen, V.C. Pham and M. Litaudon, J. Nat. Prod., 71, 2057 (2008).

124. I. Jantan, I.A. Rafi and J. Jalil, Planta Med., 67, 466 (2001)

125. F.R. Chang, J.L. Wei and C.M. Teng, J. Nat. Prod., 61, 1457 (1998)

126. F.R. Chang, Y.C. Chao and C.M. Teng, J. Nat. Prod., 61, 863 (1998).

127. K. Jenett-Siems, C. Kraft, K. Siems, et al., Sipaucins A-C, Phytochemistry, 63, 377 (2003)

128. J.J. Chen, Y.L. Chang and C.M. Teng, Planta Med., 66, 251 (2000).

129. Y. Nishiyama, M. Moriyasu and M. Ichimaru, Phytochemistry, 65, 939 (2004).

130. N.Y. Yang, N. Xie and F. Zhi, Res. Tengchun Alkaloid Compon., 30, 171 (1999).

131. M. Kubo, H. Matsuda and K. Tokuoka, Biol. Pharm. Bull., 17, 262 (1994)

132. X.H. Xu, Z.T. Wang and G.D. Yu, J. China Pharm. Univ., 33, 483 (2002)

133. S. Philipov, N. Ivanovska and R. Istatkova, Pharmazie, 55, 688 (2000).

134. C. Stévigny, S. Block and M.C. De Pauw-Gillet, Planta Med., 68, 1042 (2002).

135. K.S. Chen, Y.C. Wu and C.M. Teng, J. Nat. Prod., 60, 645 (1997)

136. K.S. Chen, Y.C. Wu and C.M. Teng, J. Nat. Prod., 60, 645 (1997)

137. T.H. Tsai, G.J. Wang and L.C. Lin, J. Nat. Prod., 71, 289 (2008).

138. S. Ayers, D.L. Zink, K. Mohn and J.S. Powell, Planta Med., 73, 296 (2007).

139. K. Morteza-Semnani, G. Amin and M.R. Shidfar, Fitoterapia, 74, 493 (2003).

140. R.L. Huang, C.C. Chen and Y.L. Huang, Planta Med., 64, 212 (1998).

141. P.H. Chuang, P.W. Hsieh and Y.L. Yang, J. Nat. Prod., 71, 1365 (2008).

142. Y. Shen, C.G. Li and S.F. Zhou, Curr. Med. Chem., 15, 1616 (2008).

143. H. Achenbach and A. Fischer, Planta Med., 63, 579 (1990).

144. L. Racková, M. Májeková and D. Kost'álová, Bioorg. Med. Chem. 12, 4709 (2004).

145. J.G. Díaz, J.G. Ruiz and W. Herz, Phytochemistry, 65, 2123 (2004).

146. T.A. Al-Howiriny, M.A. Zemaitis and F.T. Lin, J. Herb Pharmacother. 2, 1 (2002)

147. Q.A. Nguyen, H.T. Van-Dufat and S. Michel, Z. Naturforsch., 57, 986 (2002).

148. T. Tanahashi, U. Su and N. Nagakura, Chem. Pharm. Bull. (Tokyo), 48 , 370 (2000)

149. Q.B. Han, B. Jiang and S.X. Mei, Fitoterapia, 72, 86 (2001).

150. A. Shafiee and A.H. Jafarabadi, Planta Med., 64, 489 (1998)

151. A. Adsersen, A. Kjølbye and O. Dall, J. Ethnopharmacol., 113, 179 (2007).

152. T.I. Gözler, Planta Med., 46, 179 (1982).

153. I.S. Chen, J.J. Chen and C.Y. Duh, Planta Med., 63, 154 (1997)

154. A.K. Kiang and K.Y. Sim, J. Chem. Soc., 4, 282 (1967).

155. A.M. Nasser and W.E. Court, J. Ethnopharmacol., 11, 99(1984).

156. Q. Zhao, Y. Zhao and K. Wang, J. Ethnopharmacol., 106, 408 (2006).

157. T.Q. Yan and Y.F. Yang, China J. Chin. Mater. Med., 29, 961 (2004).

158. S.S. Lee and R.W. Doskotch, J. Nat. Prod., 59, 738 (1996).

159. G.L. Zhang, W.E. Pan and S.L. Peng, Acta Pharmacol. Sin., 25, 604 (1990).

160. G. Cosar, H. Bilgehan and T. Gözler, Mikrobiyol Bull.,15, 105 (1981)

161. J. Liao, W.Z. Liang and G.S. Tu, China J. Chin. Mater. Med., 19, 612 (1994).

162. H.M. Chang, A.M. El-Fishawy and D.J. Slatkin, Planta Med., 50, 88 (1984).

163. K.V. Rao and T.L. Davis, J. Nat. Prod., 45, 283 (1982).

164. I.S. Chen, J.J. Chen and I.L. Tsai, Planta Med., 61, 537 (1995).
165. J.Y. Zhou, X.J. Tong and W.Y. Lian, Planta Med., 57, 156 (1991). 166. I. Válka, D. Walterová and M.E. Popova, Planta Med., 51, 319 (1985). 167. J.J. Chen, Y.L. Chang and C.M. Teng, Planta Med., 67, 593 (2001).

168. J.J. Chen, T. Ishikawa and C.Y. Duh, Planta Med., 62, 528 (1996).

169. M. Velcheva, H. Dutschewska, G. Samuelsson, Acta Pharm. Nord., 4, 57 (1992).

170. S.S. Lee and R.W. Doskotch, J. Nat. Prod., 62, 803 (1999).

171. C. Stévigny, M.C. Wautier and J.L. Habib Jiwan, Planta Med., 70, 764 (2004).

172. H. Dutschewska, B. Dimov and V. Christov, Planta Med., 45, 39 (1982).

173. G.L. Zhang, G. Rücker, E. Breitmeier, Planta Med., 64, 165 (1998).

174. L.A. Mitscher and A. Al-Shamma, Foreign Med. Vol. Pharm., 6, 326 (1981).

175. M.K. Pyo, H.S. Yun-Choi and Y.J. Hong, Planta Med., 69, 267 (2003).

176. B. Akendengue, E. Ngou-Milama and F. Roblot, Planta Med., 68, 167 (2002).

177. A.J. Al-Rehaily, M.H.M. Sharaf and M.A. Zemaitis, J. Nat. Prod., 62, 146 (1999)

178. N. Kashiwaba, M. Ono and J. Toda, J. Nat. Prod., 63, 477 (2000).

179. Y.T. Di, H.P. He and C.S. Li, J. Nat. Prod., 69, 1745 (2006).

180. M.J. Tang, J. Zhao and X. H. Li, China J. Chin. Mater. Med., 29, 390 (2004).

181. J.H. Guh, F.N. Ko and S.M. Yu, Eur. J. Pharmacol., 279, 33 (1995).

182. B. Indra, K. Matsunaga and O. Hoshino, Eur. J. Pharmacol., 445, 21 (2002).

183. C.M. Teng, S.M. Yu and F.N. Ko, Br. J. Pharmacol., 104, 651 (1991).

184. S. Chuliá, J. Moreau and E. Naline, Br. J. Pharmacol., 119, 1305 (1996).

185. F. Orallo, Planta Med., 69, 135 (2003).

186. M.R. Mustafa and F.I. Achike, Acta Pharmacol. Sin., 21, 1165 (2000).

187. Y. Madrero, M. Elorriaga and S. Martinez, Br. J. Pharmacol., 119, 1563 (1996).

188. S. Martinez, Y. Madrero and M. Elorriaga, Life Sci., 64, 1205 (1999).

189. B. Indra, K. Matsunaga and O. Hoshino, Eur. J. Pharmacol., 437, 173 (2002).

190. B. Indra, T. Tadano and O. Nakagawasai, Life Sci., 70, 2647 (2002).

191. F. Orallo, Planta Med., 70, 117 (2004).

192. B. Indra, T. Tadano and O. Nakagawasai, Life Sci., 70, 2647 (2002).

193. B. Indra, K. Matsunaga and O. Hoshino, Can. J. Physiol. Pharmacol., 80, 198 (2002).

194. B.N. Zhou and D.G. Kingston, Planta Med., 69, 867 (2003).

195. S.H. Woo, N.J. Sun and J.M. Cassady, Biochem Pharmacol., 57, 1141 (1999).

196. N. Santanam, M. Penumetcha and H. Speisky, Atherosclerosis, 173, 203 (2004).

197. P. Kringstein and A.I. Cederbaum, Free Radic. Biol. Med., 18, 559 (1995).

198. Y.Y. Jang, J.H. Song and Y.K. Shin, Pharmacol. Res., 42, 361 (2000).

199. Y.C. Youn, O.S. Kwon and E.S. Han, Biochem. Pharmacol., 63, 495 (2002).

200. L. Milián, R. Estellés and B. Abarca, Chem. Pharm. Bull. (Tokyo), 52, 696 (2004)

201. A. Ubeda, C. Montesinos and M. Payá, Free Radic. Biol. Med., 15, 159 (1993)

202. E. Hisatomi, M. Matsui and K. Kubota, J. Agric. Food. Chem., 48, 4924 (2000).

203. L.A. Martínez, J.L. Ríos and M. Payá, Free Radic. Biol. Med., 12, 287 (1992).

204. B.K. Cassels, M. Asencio and P. Conget, Pharmacol Res., 31, 103 (1995).

205. S.M. Yu, C.C. Chen and F.N. Ko, Biochem. Pharmacol., 43, 323 (1992).

206. C.M. Teng, C.M. Hsueh and Y.L. Chang, J. Pharm. Pharmacol., 49, 706 (1997).

207. F.R. Chang, Y.C. Chao and C.M. Teng, J. Nat. Prod., 61, 863 (1998)

208. I.S. Chen, J.J. Chen and I.L. Tsai, Planta Med., 61, 537 (1995).

209. E.M. Sobarzo-Sánchez, J. Arbaoui and P. Protais, J. Nat. Prod., 63, 480 (2000).

210. M. Asencio, B. Delaquerrière and B.K. Cassels, Pharmacol. Biochem. Behav., 62, 7 (1999).

211. F. Loghin, A. Chagraoui and M. Asencio, Eur. J. Pharm. Sci., 18, 133 (2003).

212. J.J. Kang, Y.W. Cheng and W.M. Fu, Jpn. J. Pharmacol., 76, 207 (1998).

213. P.A. Erhardt and T.O. Soine, J. Pharm. Sci., 64, 53 (1975).

214. S.K. Bhattacharya, R. Bose and P. Ghosh, Psychopharmacology (Berl)., 59, 29 (1978) 
215. W.E. Fantegrossi, C.L. Kiessel and P.T. Leach, Psychopharmacology (Berl.), 173, 270 (2004)

216. R. González-Cabello, H. Speisky and R. Bannach, J. Invest. Allergol. Clin. Immunol., 4, 139 (1994).

217. S. Philipov, N. Ivanovska and P. Nikolova, Pharmazie, 53, 694 (1998).

218. N. Ivanovska, M. Hristova and S. Philipov, Pharmacol Res., 41, 101 (2000).

219. N. Ivanovska and S. Philipov, Methods Find Exp. Clin. Pharmacol., 19, 579 (1997)

220. J. Boustie, J.L. Stigliani and J. Montanha, J. Nat. Prod., 61, 480 (1998).

221. C.F. Zhang, N. Nakamura and S. Tewtrakul, Chem. Pharm. Bull. (Tokyo), 50, 1195 (2002).

222. M. Eltze, T. Grebe and M.C. Michel, Eur. J. Pharmacol., 443, 151 (2002).

223. F. Orallo and A.F. Alzueta, Planta Med., 67, 800 (2001).

224. M.D. Ivorra, F. Martinez and A. Serrano, J. Pharm. Pharmacol., 45, 439 (1993).

225. W.Y. Chen, F.N. Ko and Y.C. Wu, J. Pharm. Pharmacol., 46, 380 (1994).

226. J.J. Kang and Y.W. Cheng, Planta Med., 64, 18 (1998).

227. H. Speisky, J.A. Squella and L.J. Núñez-Vergara, Planta Med., 57, 519 (1991)
228. Y.Q. Zhao, G.R. Li and D.Z. Zhang, Zhongguo Yao Li Xue Bao, 12, 345 (1991).

229. M.L. Young, M.J. Su and M.H. Wu, Br. J. Pharmacol., 113, 69 (1994).

230. M.J. Su, Y.C. Nieh and H.W. Huang, Naunyn2Schmiedeberg's Arch. Pharmacol., 34, 422 (1994).

231. S. Ayers, D.L. Zink and K. Mohn, Planta Med., 73, 296 (2007).

232. V.K. Agnihotri, H.N. ElSohly and S.I. Khan, Phytochemistry, 10 (2008).

233. A. Morello, I. Lipchenca and B.K. Cassels, Comp. Biochem. Physiol. Pharmacol. Toxicol. Endocrinol., 107, 367 (1994).

234. J. Hung, J. Castillo and G. Jiménez, Spectrochimica Acta A: Molecul. Biomolecul. Spectrosc., 59, 3177 (2003).

235. N. Backhouse, C. Delporte and M. Givernau, Agents Actions, 42, 114 (1994).

236. M. Gotteland, I. Jimenez and O. Brunser, Planta Med., 63, 311 (1997).

237. I.L. Tsai, Y.F. Liou, S.T. Lu, Gaoxiong Yi Xue Ke Xue Za Zhi, 5, 132 (1989).

238. Q. Paulo Mde, J.M. Barbosa-Filho and E.O. Lima, J Ethnopharmacol., 36, 39 (1992). 